\title{
Multiple imputation via chained equations for elastic well log imputation and prediction
}

\author{
Antony Hallam \\ (Institute for GeoEnergy Engineering, Heriot-Watt University, United Kingdom) \\ Debajoy Mukherjee
}

(Department of Chemical Engineering, Indian Institute of Technology (IIT) Kharagpur, West Bengal 721302, India)

Romain Chassagne

(Institute for GeoEnergy Engineering, Heriot-Watt University, United Kingdom)

Keywords: MICE, well log imputation, machine learning, elastic log prediction

This manuscript has been submitted for publication in Computers and Geosciences. Please note that peer review for this manuscript is pending and that is has yet to be accepted for publication. Subsequent versions of this manuscript may have slightly different or reduced content to meet publication guidelines. If accepted, the final version of this manuscript will be available from the publisher and a DOI link will be provided. Please feel free to contact the corresponding author; we welcome feedback. 


\title{
Multiple imputation via chained equations for elastic well $\log$ imputation and prediction ${ }^{\star}$
}

\author{
Antony Hallama,*, Debajoy Mukherjee ${ }^{\mathrm{b}}$, Romain Chassagne ${ }^{\mathrm{a}}$ \\ ${ }^{a}$ Institute for GeoEnergy Engineering, Heriot-Watt University, United Kingdom \\ ${ }^{b}$ Department of Chemical Engineering, Indian Institute of Technology (IIT) Kharagpur, West \\ Bengal 721302, India
}

\begin{abstract}
Well logging is an essential component in the petroleum industry for developing a proper understanding of the subsurface geology and formation conditions. Unfortunately, the measurements are rarely complete and missing data intervals are common due to operational issues or malfunction of the logging device. Therefore the imputation of missing data from down-hole well logs is a common problem in subsurface workflows. Recently, many different approaches have been utilised but they are often manual or generalise poorly. Machine learning has reignited interest in this field with promises of a more generic and simpler approach. We explore whether the chaining of machine learning for mutli-log imputation improves results by overcoming disparities in the patterns of missing data. We will focus this work on the elastic logs of compressional (DT) and shear (DTS) sonic along with the bulk density (RHOB).

Keywords: MICE, well log imputation, machine learning, elastic log prediction

${ }^{\star}$ This manuscript has been submitted for publication in Computers and Geosciences. Please note that peer review for this manuscript is pending and that is has yet to be accepted for publication. Subsequent versions of this manuscript may have slightly different or reduced content to meet publication guidelines. If accepted, the final version of this manuscript will be available from the publisher. A DOI link will be provided. Please feel free to contact the corresponding author; we welcome feedback.

* Corresponding author

Email addresses: arh5@hw.ac.uk (Antony Hallam),

debajoymukherjee98.iitkgp@gmail.com (Debajoy Mukherjee), r.1.chassagne@hw.ac.uk (Romain Chassagne)
\end{abstract}

Preprint submitted to Elsevier

May 10, 2021 


\section{1. Introduction}

$2 \quad$ Well $\operatorname{logs}$ are digital measurements acquired during the drilling of petroleum

3 wells (Tittman, 1986). They are an important source of information for subsurface

4 specialists who utilise the data to guide exploration and development activity.

5 The logs are acquired by lowering or conveying a number of specialised tools

6 along the well bore whereby measurements are recorded periodically, typically

7 on a scale of approximately $10 \mathrm{~cm}$. Measurements cover a range of physical

8 properties related to both the rock and formation fluid surrounding the well bore

- and are typically complimentary with some lithology dependent relationships

10 between them. Unfortunately for a variety of mechanical and commercial reasons

11 which are explored further in section two, well log suites for any given well are

12 rarely complete and strategies are required to overcome gaps in the data to 13 facilitate further analysis and subsurface workflows.

14 For geophysical analyses (out interest), the elastic logs of compressional (DT) 15 and shear (DTS) sonic along with the bulk density (RHOB) are of particular 16 interest. Whilst related to each other through the framework and composition of

17 the measured rock they offer distinct information important to linking seismic

18 data with the earth. Our focus in this paper is the accurate imputation of

19 these elastic well logs. In downstream geophysical workflows, continuous logs

2о are important for seismic inversion, velocity modelling, pressure prediction and

21 synthetic well ties. Gaps in the elastic logs can compromise and complicate the 22 quality and conclusions of workflows used to inform such studies.

23 Imputation of elastic well logs has been approached using many methods.

24 Typical techniques may include manual editing with hand-drawn values, donor

25 log splicing, linear interpolation, local or lithology based mean-value fill, singular

26 log-log regressions and empirical relationships (e.g. Gardner et al. (1974) for

27 sonic and density and Greenberg and Castagna (1992) for compressional and

28 shear sonic). In simple cases with single wells and logs many of these approaches

29 are sufficient. There are however limitations, and often a degree of subjectivity

зо and interpretation required as the methods break down when applied to larger 
databases of wells, or when substantial gaps exist in the logs. Quantitative methods are required in such cases and empirical relations or numerical rock models are often employed but these are sensitive to inherent model assumptions that introduce bias error. To overcome these limitations, models can become increasingly complex and rigid as additional model constraints and variables are added to handle variations due to depth, lithology and fluid content.

The recent explosion in data science methods and availability of large amounts of well log data present an opportunity to use a more automated statistics based approach which simplifies the imputation process, improving both accuracy and turnaround. The application of machine learning methods to well log imputation or prediction and with geosciences in general is not new (Dramsch, 2020). For example, commercial applications of earlier machine learning algorithms (artificial neural networks (ANN) and radial basis functions) have been used to predict logs from seismic data and attributes (Hampson et al., 2001; Russell et al., 2003) have existed for nearly 20 years. More recently, Lopes and Jorge (2018) applied log imputation and prediction to a data set of 1026 north sea wells using a variety of methods including Bayesian Ridge Regression (BRR), Artificial Neural Networks (ANN) and decision trees. Efforts were made to generate training gaps in the data that mirrored the observed gaps in the data set. Decision tree ensembles (Random Forrest and Gradient Boosted Trees) were found to outperform all other methods.

Random forest $(\mathrm{RF})$ methods have proved popular in multiple well related use cases. Hegde and Gray (2017) applied RF to a drilling efficiency optimisation task using only surface measurements and demonstrated that offset wells could be used to improve the drilling rate over short intervals. Feng et al. (2021) applied RF to a DTS prediction tasks on the Volve data set using a quantile approach to estimate output uncertainty.

Neural network (NN) methods have also proved popular with researchers. Churikov and Grafeeva (2018) explore the prediction of the gamma-ray log using NN, comparisons were made with linear interpolation with favourable results for the NN over large gaps. There have also been attempts to apply 
deep NN methods and composite methods to log prediction problems. Jian et al. (2020) uses an ensemble learning machine combining 5 common ML algorithms including DNN in a density log prediction task. Ensemble learning machines are designed to overcome limitations in the algorithms used by combining the results of each method. The DNN was outperformed by gradient boosted tree algorithms both for accuracy and performance (DNN required three orders of magnitude more tainging time). The authors concluded that insufficient training data was available to make DNN more competitive. Ghaithi (2020) experienced similar problems with a feed forward neural network where lithological zones which contained insufficient data either over or under predicted the truth.

Comparisons of different ML algorithms with empirical regression models for predicting shear sonic logs are made by Bukar et al. (2019). The authors concluded that Gaussian Process regression was superior to other methods and importantly, that it could account for variations in fluid saturation. The fluid content of a rock is an important aspect of well logging and fundamental to downstream subsurface analyses. Similar results were found by Brown et al. (2020), where the downstream petrophysical analysis workflow was bypassed by training gradient boosted tree algorithms to predict petrophysical logs directly such as water saturation and porosity. Training data in this case must include expertly curated petrophysical logs with special attention paid to preprocessing of the input logs.

The application of certain types of machine learning can also have additional benefits. Diaz and Zadrozny (2020) apply radial basis functions (similar to BRR) to impute gaps in the gamma-ray log. This Bayesian approach incorporates the posterior imputation uncertainty providing confidence measures on the output. This additional information was used in subsequent geostatistical modelling. The approach used by Feng et al. (2021) for quantile RF is similar, but provides a measure of the ensemble prediction variance.

Many of the discussed literature propose approaches to feature engineering, a process for data improvement or augmentation to improve results. Churikov and Grafeeva (2018) applied smoothing to the logs to reduce noise going into 
their NN models. Ghaithi (2020) found that including additional non-tool logs such as depth improved the overall fit of the data while Hegde and Gray (2017) suggest that derivative (deterministic) logs created by domain experts improved results.

Scaling and standardisation of the data is also an important feature engineering pre-processing step. Feng et al. (2021) and Churikov and Grafeeva (2018) transform logs with a logarithmic response (such as resistivity) to a more linear scale. Most authors also mention removing bias and scale differences between input logs. Finally, Feng et al. (2021) tests feature space redundancy reduction using measures of feature importance and principle component analysis.

In general, all of these examples have only considered machine learning approaches that train for a single target log. This approach honors the input data but we hypothesise that imputed log values can become more accurate when input features are also imputed. Most machine learning algorithms also require the input feature set to be complete. Due to the nature of missing values in well logs this would significantly limit the sections of the well where models can be trained and applied. By approaching imputation of all input features simultaneously a greater portion of the well can be imputed for more accurately. Such an approach would also enable a wider variety of prediction models to be adopted, not just those that can handle missing values in the input.

To explore this idea, this study applies the multiple imputation via chained equation (MICE) algorithm (van Buuren and Groothuis-Oudshoorn, 2011). MICE is an imputation algorithm that does not specify a prediction strategy, thus within the framework of MICE we test three common machine learning predictors (gradient boosted trees, bayesian ridge regression and k-nearest neighbours). Our study explores how multi-stage imputation might improve the prediction results in diverse data sets when compared with single stage or direct prediction.

The method/s will be tested first by standard ML evaluation techniques (accuracy scores of mean square error, absolute error, explained variance and Pearson's $R^{2}$ factor), using a blind multi-well test where known values will be predicted by the model trained on other wells. 
The adoption of machine learning requires the introduction of new terminology unfamiliar to a typical well logging audience. Within this paper we refer to logs and features. Feature is a common term use to describe vector inputs to machine learning algorithms. For our purposes, features have the same dimension size as the input logs, however feature engineering of the logs may be used to scale, transform or modify the input to improve prediction accuracy or to suit prediction algorithm limitations. If features are used in place of the input logs, the feature engineering must be reversible for the $\log$ to be recovered after imputation. An imputer or imputation strategy refers specifically to a workflow or algorithm used to fill missing values. Algorithms or models termed predictors are a component part of any imputation workflow, either an empirical model or in the case of this study, machine learning. Imputation workflows typically require at least one or more prediction models for each log being imputed.

\section{Data Imputation and Prediction}

The fundamental aim of data imputation is to accurately predict values which are missing from a data variable or data set to provide complete input variables for further analysis. When performing imputation it is important to consider the style or character of a data sets missing values as this can impact the imputation strategy and prediction algorithms used. In the context of data missingness, character refers to the overall fraction of missing values, the distribution of the missing values between features, the continuity of the missing values and correlation of missing values between features. The more a data set matches these criteria, the more difficult accurate and robust imputation of the data set will be. The reasons for missing values are generally specific to the experiment being performed but are commonly due to experimental restrictions, mistakes or failures.

There are many approaches to estimating missing values which range from simple mean or median estimates, to regression models. More recently machine learning algorithms have been gaining in popularity in the geosciences (Dramsch, 2020). The efficacy of any method depends upon the type of data being imputed, 
the quality and distribution of the known values and the complexity of the imputation model. It is important to consider the data being imputed, the way in which the imputed data will be further studied and analysed and the data set as whole when selecting an imputation strategy.

If the imputation model relies upon a stochastic approach - as most machine learning does - the robustness of the predictions will rely upon the completeness of distribution sampled by the available data. Stochastic style imputation models can generally not be used to predict unknown or unseen measurements. The available measurements should also be relatively free of noise, excluding outliers and bad or improper data which will skew the input distribution.

The missingness character of well logs is generally related to processes by which logs in any given well are acquired. From a macro perspective logs may be partially or entirely absent from a well, and at smaller scales, gaps occur for operational reasons or due to log editing and quality control processes. The reasons for not recording data are varied but commonly include; commercial considerations (cost and time), logging tool availability, mechanical failures of the measurement tools and drilling equipment and geometric constraints within the well bore and along the tool string. Bad data often occurs in cased hole logging, or due to the breakdown of borehole conditions (which is usually lithology dependent) and tool failures. Importantly for imputation, much of this missing data is not random but blocky and regular. As examples; the shallow sections of well bores, particularly in the Oil and Gas sector are not readily logged by all tools, also, casing points which interrupt logging often occur in the same formations due to borehole engineering design restrictions and lithologically unstable formations may result in a higher data failure rate. The cumulative effect of these problems can cause missing data to be non-random.

Well logs must undergo a large number of manual corrections which merge, depth align and normalise separate tool logging runs within wells. Logs are also often normalised across large well databases to correct for differences due to tool models and calibrations. Without these quality control steps and manual edits for bad or inconsistent data, subsequent tasks including imputation and 
prediction may become compromised.

Finally, there are aspects of well logs and geology that strongly affect the capability of regression techniques. The geology can change rapidly in the vertical direction with each geological zone having distinct logging properties and characteristics. Over larger distances these zones can also change laterally as chronostratigraphic layers with common names differ lithologically. Logging tools also measure at different scales and distances away from the well bore, and may not necessarily be measuring the same volume of the formation. Single lithologies can also vary with depth, leading to non-stationary data over the depth range of a well. Wells intersecting common geology at distinctly different depths can have significantly different log-to-log relationships that need to be accounted for by any prediction model.

Multiple model types are suitable to well log imputation. Due to the quantity of data, regression using empirical models or user derived relationships are common. These models are often restricted to single lithological zones or localised fields and cumbersome to calibrate and implement. They are also generally one-pass models, relying upon the coincident available data to calculate trends. We use this paper to explore the application of iterative multiple imputation to well logs where imputed values are subsequently used to improve the estimates of other missing data until a convergence tolerance or iteration limit is obtained.

\section{1. $M I C E$}

Multivariate imputation of chained equations (MICE) (van Buuren, 2007, van Buuren and Groothuis-Oudshoorn, 2011) is a multi-feature, prediction model agnostic imputation strategy. MICE supposes that the output of prediction models used for imputation can be improved by chaining together a series of imputation models for all input features (well logs). With each iteration of the complete feature set, the accuracy of the imputed values improves as bias in the prediction models is reduced (Azur et al. 2011). The order in which imputation proceeds can be random or based upon the completeness of features (Raghunathan et al., 2001; Varoquaux et al., 2015). Iteration is ceased when 
imputation predictions converge towards a stable solution.

The MICE imputation strategy offers advantages in data sets with large amounts of randomly missing data. Here, random implies that the missingness of a value is not correlated with the value itself. By imputing for all values the MICE algorithm should improve predictors that rely upon complete samples in the input data set for training and prediction. In this way, partially complete sample points may still contribute towards the imputation predictions. MICE also avoids complications arising from joint modelling techniques where multivariate distributions must be specified. MICE does this by imputing for each variable individually.

Apart from the requirement that data should be Missing At Random (MAR), there are few other assumptions in the MICE algorithm (Algorithm 1). The freedom to choose a prediction method leaves the user to implement a method that suits the data domain or distribution. Indeed custom predictors of constraints may be introduced to improve results (Azur et al., 2011; Raghunathan et al. 2001). Generalised prediction models are generally preferred due to the lack of user interaction or parameterisation required. In this paper we explore the performance of three well known prediction models, Bayesian Ridge Regression (BRR), K-Nearest Neighbours (KNN) and Gradient Boosted Trees (GBT).

As an imputation workflow, MICE appears to be well suited to imputing well logs. Although missing values in wells often occur in blocks or commonly are entirely missing. If the data set is sufficiently large however, it may still meet the MAR requirement. In this study, our focus on machine learning and stochastic predictors limits the accuracy where logs cannot be characterised through relationships with other input logs or features.

Finally, most predictors cannot sample beyond the known inputs (e.g. extrapolation is not possible). The input data must contain information sufficient to cover the distribution of the missing values to be impacted. 


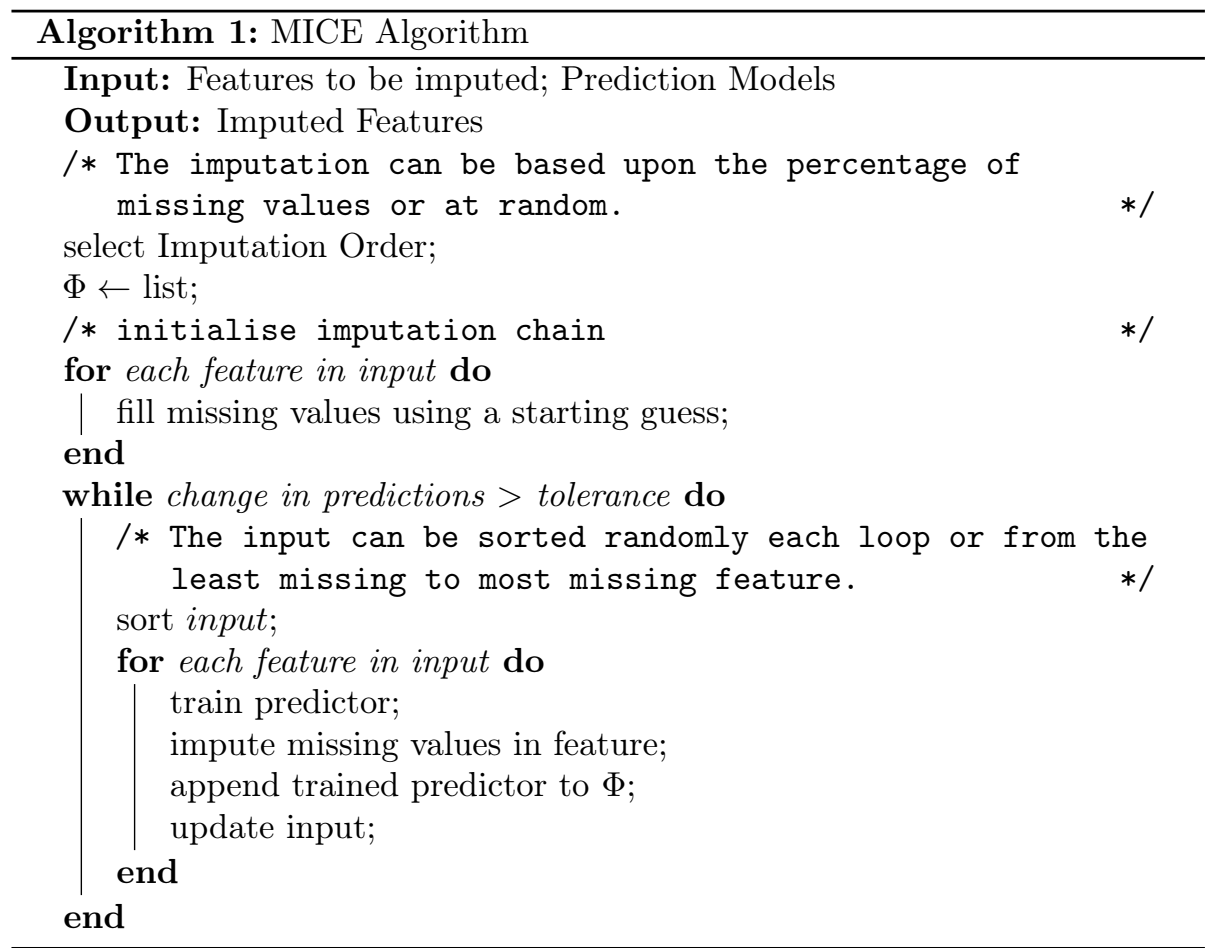

\subsection{Predictor 1: Bayesian Ridge Regression}

Bayesian Ridge Regression (BRR) can be described as a Bayesian extension of the popular Support Vector Machine (SVM) regression algorithm (Tipping, 2001). Both methods approximate the output from the training data by solving for the coefficients of a weighted sum of basis functions. Constraints are applied to the solution which penalise the number of non zero basis functions preventing over-fitting of the noise which results in good generalisation properties. BRR differs from SVM by introducing Bayesian inference to explicitly model the noise within the data, which is assumed to have a Gaussian distribution. BRR tends to require fewer basis functions, produces probabilistic estimates of the solution and has fewer restrictions on the basis function kernel (Tipping, 2001).

The implementation used by this paper (Varoquaux et al., 2015) maximises the marginal likelihood of the hyperparameters controlling the smoothness of the output and the shape of the prior noise Gaussian distribution to fit the data. 
Although a probabilistic solution is returned, the predicted value will be taken as the highest probability point of the output distribution.

\subsection{Predictor 2: K-Nearest Neighbours}

K-Nearest Neighbours (KNN) regression, is a technique that lazily models a function by considering a distance weighted average of a neighbourhood of known values (Dubey and Pudi, 2013, James et al., 2013, Poloczek et al., 2014). The regression solution is only an approximation to the true function but the method is well suited to multi-dimensional problems such as well logs. Unlike $\mathrm{BRR}, \mathrm{KNN}$ methods are non-parametric, and the distribution of the output is not prescribed by a specific model or prior distribution. This can be helpful when input logs are discontinuous or classification based such as for geological zones.

Key considerations when using KNN regression are related to the inputs and hyper parameters of the algorithm. KNN regression benefits from data standardisation (zero mean unit standard deviation) of the input data because the distance metrics are strongly affected by the input magnitude. The best value for $k$, the number of nearest neighbours, is often found experimentally. A larger value of $k$ will tend to suppress noise but could blur the boundary between values.

With a large number of input features, points become increasing equidistant and the discrimination power of KNN can begin to degrade. To overcome this, pre-processing through dimensionality reduction may be helpful, such as principle component analysis to remove or consolidate redundant features.

There are multiple techniques to efficiently generate the neighbourhood of points. The naive, brute force approach is often supplemented for tree based algorithms where a setup phase is used to calculate cumulative distance between points as a network for easier retrieval later (Varoquaux et al., 2015).

The implementation of KNN utilised by this study uses an equal weighting between input features and measures distance via the euclidean distance between points. The method is also unsupervised, with the number of neighbourhoods 
determined by the algorithm.

\subsection{Predictor 3: Gradient Boosted Trees}

Gradient Boosted Tree (GBT) algorithms are a recent and relatively successful evolution of the decision tree machine learning algorithm. In a decision tree, input data is spread from the starting root through branches to decision split points whereupon it is routed into two new branches based upon a single logical operator. The operator used at each split can vary but generally aims to maximise the information gain or prediction accuracy after the split. Decision trees can continue to grow until they have a single data-point in each leaf but typically are limited to a maximum number of splits or depth. A single decision tree is generally a poor predictor for any model and they are termed weak learners.

Gradient boosting first used in AdaBoost (Freund and Schapire, 1997) seeks to transform decision trees from weak learners to strong learners by retraining recurrently against an objective and regularisation function. Standard objective functions can be solved using gradient descent style methods following the derivative of the error until a minimum is reached. Decision trees have no such derivative and as a result additive training (boosting) is used. In boosting the predictive tree functions are added after each training round until optimisation is achieved ensuring each decision tree is optimal prior to being included in the algorithm.

A single decision tree alone, even a gradient boosted tree still performs relatively weakly as a predictive tool. Predictive accuracy is greatly enhanced by employing ensemble methods. Random forests are a well known ensemble method that leverage a variety of statistical tools to create multiple unique decision trees. The accumulative results of multiple trees generates a significantly more accurate predictive model. To introduce tree diversity during training random forest algorithms randomly sample subsets of the input data set using bootstrap aggregation (bagging via representivity and independence). Data subsets will randomly sample both the feature space (dropping features) and the sample space (dropping samples) to generate a broad range of unique decision trees 
within the forest.

There are many benefits to GBT and decision tree methods. Decision trees often scale well with data input, are fast and have a simplicity that does away with much of the pre-processing needs. They also avoid implicit regression models and are non-parametric (distribution agnostic) relying only on a logical (greater than or less than) operator at each decision branch. Decision trees also have transparency and can be inspected easily compared with other machine learning approaches such a neural networks. Finally, decision trees allow for manual tuning of the bias and variance error trade-off through their hyperparamters.

GBT also benefit from being able to accept missing values as input. Decision trees achieve this by considering whether a value is missing or not as the logical split, solving the remainder of the problem as normal. For this reason we include testing of direct or single pass imputation with GBT (referred in the rest of the document as D-GBT) as well as in a MICE approach.

There are many popular implementations of GBT available, but we utilise LightGBM within this study (Ke et al., 2017).

\section{Test Data, Preprocessing and Conditioning}

This study utilises two distinct well log data sets. The two data sets were selected due to their open availability, their preconditioning, comprehensive labelling and curation by subject matter experts. The two data sets also cover distinct spatial scales representing an increasing level of geological diversity and subsequent imputation difficulty.

Exact information about the preconditioning applied to the two data sets is not available but examination of the logs suggests the following processes have been applied and conditions have been met.

1. Logs from multiple tools runs have been depth aligned and merged to generate a single log for each measurement type.

2. Logs have been depth aligned using key markers. 
3. Significant noise due to borehole conditions, logging through casing and other errors have been edited from the data. For Volve, this was performed by the authors.

4. Common logs (e.g. gamma-ray) have been normalised to account for variations in tool models and calibration settings.

5. Litho-stratigraphic interpretations provided with the training data are accurate and complete.

Appropriate preconditioning of the data prior to imputation is an important step. Performance of the imputation model, will be heavily dependent upon the quality of the input data.

\subsection{Volve}

The Volve data set comprises 20 wells from a single oil field in the Norwegian North Sea. The data was released by Equinor in 2018 as part of a complete field data set. The drilling of the wells spans approximately 20 years with initial exploration and appraisal wells often having more complete log suites (Hallam et al. 2020). In offshore field well log data sets, wells drilled later often have reduced logging programs due to commercial considerations and Volve follows this trend. The production wells drilled from 2007 on-wards are less likely to contain full logging suites and in particular, elastic logs.

Prior to any machine learning the Volve logs were first inspected in a standard petrophysics package. The logs were analysed to identify sections which were either interpolated, incorrectly recorded or of general poor quality. Data determined to be unsuitable for model training was removed. The log data was then exported in LAS format to be loaded into Python for further analysis. The Equinor data set provided both raw and processed logs (logs with merged runs, depth shift corrections and other petrophysical quality control) as well as petrophysical interpretations (e.g. formation water saturation, total porosity). Apart from the formation tops, petrophysical interpretation logs were not included in this study. 
The deepest part of the logs cover the reservoir and surrounding geological formations. For the remainder of this study the logs recorded shallower than the top Ty Formation have been removed. The total number of samples available for learning is 172167 . Within the filtered data set, log coverage varies greatly; Gamma-ray (GR) has almost no missing values for the zones of interest whilst shear-sonic (DTS) has over 60\% missing values (Figure 1(a)). The three key elastic logs, density, compressional sonic and shear sonic have coverage of $80 \%$, $60 \%$ and $35 \%$ respectively.

\subsection{Force 2020 Well Log Machine Learning Data set}

A second larger test data set containing more than 90 wells from offshore Norway has been used to test the generalisation of this imputation methodology beyond closely related geological areas. The data set was originally created for the Force 2020 Machine Predicted Lithology (F2020) (Bormann et al., 2020) competition. Imputation of the missing values was a key step towards the objective of geological facies prediction.

The F2020 data set is provided in a pre-created train and test split. The split has been created arbitrarily by the data provider separating complete wells from training data. So called blind well testing in common in subsurface geosciences and are considered to offer a more realistic measure of the predictive capacity of models. The 10 test wells are evenly distributed within the input data, but the percentage of missing values per input log can vary dramatically between the training and test sets (Table 1 ).

The total size of the F20 data set is 1,307,118 samples with approximately $10.5 \%$ belonging to the test set. There are a greater number of logs (features) made available with the F2020 data set which has missing values in the training data set per feature ranging from $0-95 \%$. The elastic logs DTC, DTS and RHOB have missing values of $6.9,85.1$ and $13.8 \%$ respectively. Note that DTC is a common alias for DT and we use it here to remain consistent with the source data.

Due to the spatial extent of the data set, some of the additional logs provided 
ption

have been included as imputation constraints, namely, the Cartesian coordinates

of the sample (X_LOC, Y_LOC) but drilling metrics and non-critical logs with a large proportion of missing values have been excluded, specifically bit size (BS), rate of penetration (ROPA), mud-weight, and spectral gamma ray (SGR).

Table 1: Data sets log description and missing summary for imputed logs.

\begin{tabular}{|c|c|c|c|c|c|c|c|}
\hline \multirow[t]{3}{*}{ Description } & \multirow[t]{3}{*}{ Type } & \multicolumn{2}{|c|}{ Log Pneumonic } & \multicolumn{4}{|c|}{ Missing (\%) } \\
\hline & & VOLVE & F2020 & Volve & Volve & F2020 & F2020 \\
\hline & & & & Train & Test & Train & Test \\
\hline Well ID & Cat & & WELL & & & 0 & 0 \\
\hline Measured Depth & Cont & & DEPTH_MD & & & 0 & 0 \\
\hline Well Head Easting & Cont & & $\mathrm{X} \_\mathrm{LOC}$ & & & 0 & 0 \\
\hline Well Head Northing & Cont & & $\mathrm{Y} \_\mathrm{LOC}$ & & & 0 & 0 \\
\hline Well Head Elevation & Cont & TVDSS & $\mathrm{Z} \_\mathrm{LOC}$ & 0 & & 0 & 0 \\
\hline $\begin{array}{l}\text { Stratigraphic Forma- } \\
\text { tion }\end{array}$ & Cat & ZONE & FORMATION & 0 & 0 & 0 & 0 \\
\hline Caliper & Cont & & CALI & & & 4 & 0.03 \\
\hline $\begin{array}{l}\text { Averate Rate of Pene- } \\
\text { tration }\end{array}$ & Cont & & ROPA & & & 54.13 & 45.5 \\
\hline $\begin{array}{l}\text { Spontaneous Poten- } \\
\text { tial }\end{array}$ & Cont & & SP & & & 24.14 & 53.35 \\
\hline Medium Resistivity & Cont & $\mathrm{RM}$ & RMED & 37.13 & 7.3 & 1.34 & 0.22 \\
\hline Deep Resistivity & Cont & $\mathrm{RD}$ & RDEP & 15 & 0.08 & 0.01 & 0 \\
\hline Density & Cont & RHOB & RHOB & 0.6 & 17.4 & 8.2 & 0.55 \\
\hline Density Correction & Cont & & DRHO & & & 10.22 & 7.44 \\
\hline Gamma-Ray & Cont & GR & GR & 0.01 & 0 & 0 & 0 \\
\hline Neutron Porosity & Cont & NPHI & NPHI & 0.7 & 0.04 & 30.41 & 13.7 \\
\hline Photo Electric Factor & Cont & $\mathrm{PEF}$ & $\mathrm{PEF}$ & 18.32 & 7.27 & 38.7 & 5.8 \\
\hline Compressional Sonic & Cont & $\mathrm{DT}$ & DTC & 19.97 & 9.49 & 4.6 & 0.55 \\
\hline Shear Sonic & Cont & DTS & DTS & 38.12 & 20.05 & 84.1 & 64.1 \\
\hline
\end{tabular}

412

413

\subsection{Log Editing, Scaling and Feature Engineering}

Log editing, transformations and scaling are necessary data cleaning processes for most imputation algorithms. Significant edits are as per the beginning of this section but scaling of logs to suit prediction algorithms is required. A common process is to scale each sample of an input $\log x_{i}$ to to a new feature $x^{\prime}$ by the mean $\mu_{x}$ and standard deviation $\sigma_{x}$ of $x$ (Sarkar et al. 2018) sometimes referred 
to as centering and scaling.

$$
x_{i}^{\prime}=\frac{x_{i}-\mu_{x}}{\sigma_{x}}
$$

This removes inherent bias due to differences in scale and ensures all features cover a common range. The scaling factors $\mu_{x}$ and $\sigma_{x}$ are stored so that, after imputation logs can be returned to their original scale.

Some logs require additional consideration prior to imputation. Resistivity logs naturally exhibit a logarithmic scale inherent to the measurements made by the logging tool. To improve the linear correlation and low end sensitivity between the resistivity and other logs, they have been transformed through a base 10 logarithm. For example the deep resistivity is transformed to the imputation feature $R E S D_{10}$ via the relationship $R E S D_{10}=\log _{10}(R E S D)$.

Categorical logs such as the Well ID and Stratigraphic Formation were transformed to numeric values through an integer encoder. For algorithms such as KNN regression, the distance between integers may affect the results. Adjacent or similar formations should therefore be given similar integer values. This is less critical for tree ensemble approaches.

We do not test the development of other features, but depending upon the data set, it may be useful to include other engineered logs such as smoothed variants of the raw input and other complimentary data types such as seismic traces or additional stratigraphic control like biostratigraphy labels. Due to the way sediments are deposited chronologically they can also be considered as a form of time-series. The algorithms applied in this study do not consider adjacency or the sequence of the data and prediction algorithms which account for a samples neighbourhood may perform better. Window based features could also be used to introduce neighbourhood information such as those offered by Christ et al. (2018) however, we suggest caution when doing this. Depending upon the size and frequency of the missing value gaps, many windowed of multi-sample based features could be difficult to compute accurately. Therefore, if attempting to use multi-sample dependent engineered features, special care should be exercised 
around missing values.

\subsection{Feature Selection}

Feature selection, the task of choosing which logs or features to include in the imputation is often a difficult task. In some cases, exhaustive testing can be carried out to determine the correlation between data or impact upon the prediction algorithm. We defer to domain knowledge of the measured logs, as well as the percentage of missing values. Very sparse logs are excluded, except for the shear-sonic which we wish to predict even if missing. We also exclude engineering or borehole specific logs (e.g. caliper) that are not directly correlated with geological measurements.

\subsection{Data Testing and Preparation}

Data sets were first split into training and test data sets based upon wells. The Volve blind test wells were F-4, F-12, F-1 \& F-15D. The testing split for the Force 2020 data set was provided with the data.

For training evaluation a further reduction of the training set was applied for each target $\log$ (DT, DTS, RHOB). This reduction created a unique training set for evaluationg the predictive capacities of models for DT, DTS and RHOB individually. This approach was taken because aggressive sub-setting of all target logs would have over-decimated the training data set. For testing, a further $30 \%$ of non-nan values were used from each target log. The data was removed in a random fashion, this contradicts the usual scenario where data is missing blockwise in logs but better meets the assumptions of the imputation models.

The high coverage of RHOB available in the Volve data set limited our ability to test the capacity for imputation when RHOB is not acquired. Thus, to augment and extend the testing of the Volve data set we introduce a block of missing RHOB values to simulation situations where all three elastic logs are missing. 


\section{Results}

In this study, the models and predicted values were evaluated twice. First via a training set, where $30 \%$ of values were reserved for validation, and subsequently by using a test set containing a selection of blind wells. Both tests are evaluated based upon metrics for accuracy and variance of the imputed values. Imputation models were then tested against varying degrees of input sparsity (retraining for each level of sparseness) ranging from 10 to $90 \%$. Sparsity to the original data set was introduced randomly to all input features.

Further analysis was then conducted on the preferred models using SHAP (Lundberg and Lee, 2017) to better understand feature importance and followed by a qualitative assessment of the predictive capacity.

\subsection{Volve Log Imputation}

Imputation of the logs has been performed with seven different prediction approaches (Table 2). Four of these use the MICE algorithm and impute features with ascending order of missingness (MICE-BRR, MICE-O-BRR, MICE-KNN, MICE-O-KNN and MICE-A-GBT) and one model uses a random imputation order each iteration (MICE-R-GBT). Changing the order of imputation is designed to test assertions by Murray (2018) that randomisation between iterations can improve sampling statistics and bias.

For a comparison against the MICE tests we also perform direct imputation using GBT (D-GBT model). This is not possible for BRR and KNN models because they cannot handle prediction when the input features are incomplete. Instead, we perform imputation using just a single pass of MICE (one imputation model per input feature, MICE-O-BRR and MICE-O-KNN). All applications of MICE utilise the mean feature value as the initial guess for missing values.

To evaluate the models, five metrics for accuracy and bias are calculated, these are the explained variance, maximum error, mean absolute error (MAE), mean squared error (MSE) and Pearson's $R^{2}$ or correlation factor. Explained variance and $R^{2}$ values range from 0 to 1 with larger values indicating greater 
Table 2: Imputation Model Descriptions

\begin{tabular}{|c|c|c|}
\hline Name & $\begin{array}{l}\text { Imputation } \\
\text { Order }\end{array}$ & Description \\
\hline MICE-BRR & Ascending & BRR using full MICE. \\
\hline MICE-O-BRR & Ascending & $\begin{array}{l}\text { BRR using MICE imputation } \\
\text { for one iteration. }\end{array}$ \\
\hline MICE-KNN & Ascending & KNN using full MICE. \\
\hline MICE-O-KNN & Ascending & $\begin{array}{l}\text { KNN using MICE imputation } \\
\text { for one iteration. }\end{array}$ \\
\hline MICE-A-GBT & Ascending & GBT using full MICE. \\
\hline MICE-R-GBT & Random & GBT using full MICE.. \\
\hline D-GBT & $\mathrm{N} / \mathrm{A}$ & $\begin{array}{l}\text { Direct imputation of missing } \\
\text { values using a single GBT } \\
\text { model, no MICE. }\end{array}$ \\
\hline
\end{tabular}

correlation between truth and predicted logs. Error metrics range from zero to infinity with smaller values indicating better performance.

Initially we consider the results from the validation sub-set (Table 3). For Volve, the explained variance and $R^{2}$ values are approximately $\geq 0.9$ except for the BRR model which performed poorly relative to KNN and GBT models. Error rates exhibited similar trends with KNN and GBT models performing consistently well.

Compared to the baseline single imputation models (Once) BRR and KNN showed only small improvements or similar performance. The exception was for the RHOB log imputations where performance degraded. The D-GBT model showed similar or slightly worse performance than MICE approaches.

Metrics calculated using the test data were significantly lower (Table 4). The explained variance and $R^{2}$ metrics dropped to between 0.6 and 0.8. Overall, error metrics also degraded increasing by $200-300 \%$.

In Figure 3 we explore the link between geological zones, which define packages of rock with similar properties (denoted by color). The correlation of the input and predicted logs for three distinct model types; MICE-R-GBT, MICE-KNN and MICE-BRR are plotted. The MICE-R-GBT results tend to show the tightest 
Table 3: Metric scores for imputed values using different imputation algorithms on the Volve validation set. Best values are highlighted in bold.

\begin{tabular}{|c|c|c|c|c|c|c|}
\hline Model & Log & $\begin{array}{c}\text { Explained } \\
\text { Variance }\end{array}$ & $\begin{array}{c}\text { Maximum } \\
\text { Error }\end{array}$ & $\begin{array}{c}\text { Mean } \\
\text { Absolute } \\
\text { Error }\end{array}$ & $\begin{array}{c}\text { Mean } \\
\text { Squared } \\
\text { Error }\end{array}$ & $R^{2}$ \\
\hline MICE-BRR & DTS & 0.78 & 5.67 & 0.26 & 0.24 & 0.78 \\
\hline MICE-O-BRR & DTS & 0.78 & 5.72 & 0.26 & 0.24 & 0.78 \\
\hline MICE-KNN & DTS & 0.9 & 6.37 & 0.14 & 0.1 & 0.9 \\
\hline MICE-O-KNN & DTS & 0.89 & 6.18 & 0.15 & 0.12 & 0.89 \\
\hline MICE-A-GBT & DTS & 0.91 & 4.94 & 0.16 & 0.1 & 0.91 \\
\hline D-GBT & DTS & 0.91 & 5.52 & 0.16 & 0.1 & 0.91 \\
\hline MICE-R-GBT & DTS & 0.92 & 4.61 & 0.15 & 0.08 & 0.92 \\
\hline MICE-BRR & DT & 0.87 & 14.3 & 0.23 & 0.14 & 0.87 \\
\hline MICE-O-BRR & DT & 0.86 & 11.27 & 0.25 & 0.14 & 0.86 \\
\hline MICE-KNN & DT & 0.92 & 3.58 & 0.15 & 0.08 & 0.92 \\
\hline MICE-O-KNN & DT & 0.90 & 3.92 & 0.17 & 0.10 & 0.90 \\
\hline MICE-A-GBT & DT & 0.93 & 3.15 & 0.16 & 0.07 & 0.93 \\
\hline D-GBT & DT & 0.93 & 3.48 & 0.16 & 0.07 & 0.93 \\
\hline MICE-R-GBT & DT & 0.94 & 3.36 & 0.15 & 0.06 & 0.94 \\
\hline MICE-BRR & RHOB & 0.63 & 19.59 & 0.43 & 0.37 & 0.62 \\
\hline MICE-O-BRR & RHOB & 0.65 & 19.6 & 0.43 & 0.34 & 0.65 \\
\hline MICE-KNN & RHOB & 0.89 & 19.91 & 0.17 & 0.11 & 0.89 \\
\hline MICE-O-KNN & RHOB & 0.90 & 19.92 & 0.17 & 0.10 & 0.90 \\
\hline MICE-A-GBT & RHOB & 0.91 & 19.24 & 0.18 & 0.09 & 0.91 \\
\hline D-GBT & RHOB & 0.91 & 19.23 & 0.18 & 0.09 & 0.91 \\
\hline MICE-R-GBT & RHOB & 0.92 & 19.48 & 0.17 & 0.08 & 0.92 \\
\hline
\end{tabular}


Table 4: Metric scores for imputed values using different imputation algorithms on the Volve test set. Best values are highlighted in bold.

\begin{tabular}{|c|c|c|c|c|c|c|}
\hline Model & $\log$ & $\begin{array}{l}\text { Explained } \\
\text { Variance }\end{array}$ & $\begin{array}{c}\text { Maximum } \\
\text { Error }\end{array}$ & $\begin{array}{c}\text { Mean } \\
\text { Absolute } \\
\text { Error }\end{array}$ & $\begin{array}{c}\text { Mean } \\
\text { Squared } \\
\text { Error }\end{array}$ & $R^{2}$ \\
\hline MICE-BRR & DTS & 0.51 & 2.2 & 0.27 & 0.12 & 0.49 \\
\hline MICE-O-BRR & DTS & 0.5 & 2.2 & 0.27 & 0.12 & 0.49 \\
\hline MICE-KNN & DTS & 0.4 & 2.76 & 0.30 & 0.16 & 0.31 \\
\hline MICE-O-KNN & DTS & 0.51 & 2.62 & 0.26 & 0.13 & 0.44 \\
\hline MICE-A-GBT & DTS & 0.6 & 2.35 & 0.28 & 0.13 & 0.43 \\
\hline D-GBT & DTS & 0.69 & 2.33 & 0.20 & 0.09 & 0.63 \\
\hline MICE-R-GBT & DTS & 0.65 & 2.32 & 0.27 & 0.13 & 0.47 \\
\hline MICE-BRR & $\mathrm{DT}$ & 0.69 & 6.55 & 0.36 & 0.32 & 0.65 \\
\hline MICE-O-BRR & DT & 0.72 & 5.54 & 0.35 & 0.28 & 0.7 \\
\hline MICE-KNN & DT & 0.72 & 3.75 & 0.36 & 0.28 & 0.69 \\
\hline MICE-O-KNN & DT & 0.66 & 3.69 & 0.40 & 0.34 & 0.63 \\
\hline MICE-A-GBT & DT & 0.78 & 4.03 & 0.31 & 0.21 & 0.77 \\
\hline D-GBT Direct & DT & 0.81 & 2.95 & 0.29 & 0.17 & 0.81 \\
\hline MICE-R-GBT & DT & 0.80 & 4.37 & 0.31 & 0.2 & 0.78 \\
\hline MICE-BRR & RHOB & 0.44 & 8.29 & 0.58 & 0.65 & 0.38 \\
\hline MICE-O-BRR & RHOB & 0.53 & 6.14 & 0.57 & 0.56 & 0.46 \\
\hline MICE-KNN & RHOB & 0.63 & 6.22 & 0.39 & 0.41 & 0.61 \\
\hline MICE-O-KNN & RHOB & 0.64 & 6.64 & 0.39 & 0.41 & 0.6 \\
\hline MICE-A-GBT & RHOB & 0.67 & 5.36 & 0.35 & 0.36 & 0.65 \\
\hline D-GBT & RHOB & 0.67 & 5.6 & 0.36 & 0.38 & 0.63 \\
\hline MICE-R-GBT & RHOB & 0.65 & 5.65 & 0.36 & 0.39 & 0.63 \\
\hline
\end{tabular}


correlation around the 1-1 line (perfect prediction). This is particularly true for the high slowness values in DTS. The MICE-BRR and MICE-KNN models both appear to under-predict DTS slowness at high values as well as RHOB in specific zones.

Overall, the D-GBT approach was the best imputer on the test data set for the three logs.

\subsection{Volve Qualitative Analysis}

Here we use a qualitative analysis to gauge the suitability of the imputed results. Metrics provide a quantitative view of the data match but the imputed and predicted values must be assessed for their believability by a geoscience professional.

The BRR model results (Figure 4) show some interesting trends. The DT log appears to be well matched even where there are a high number of missing feature samples (between 10,000 and 15,000). Where the values for DT are high however, BRR appears to greatly over predict the DT log. A limited number of DTS values for testing were available but where they exist the MICE-BRR model seems to consistently under predict the slowness. There may be some bias from the other wells used for training against these samples. The RHOB predictions appear overly smooth compared with the known values, and they become inaccurate where the PEF and DRHO logs are absent.

The MICE-KNN model (Figure 4) matches the low frequency trends in the data but appears more prone to noise overall than the other models. The MICE-KNN model also returned no extrema beyond the models known values due to it's averaging approach. Compared with MICE-BRR, the MICE-KNN model better honours the known RHOB values without over smoothing but still struggles to eliminate the bias where PEF and DRHO are missing.

Both of the MICE-X-GBT models perform well, overall the predictions appear superior to MICE-BRR and MICE-KNN. The presence of the RHOB bias when missing DRHO and PEF suggest an inherent limitation between the available input features and the output. 
Although the D-GBT model performed well in the metrics test we can build an appreciation for the limits of the method when analysing the qualitative results. Where the deliberate absence of any elastic values has been introduced between samples 25,000 and 31,000 (Figure 4) the quality of the prediction begins to break down for both RHOB and especially for DTS. The MICE implementation of GBT tends to outperform D-GBT in these situations where directly imputing for DTS from non-elastic logs is more difficult. It appears that sequential imputation tends to improve the overall prediction result in these extreme cases of many missing values. DTS for example with D-GBT imputation has a mean squared error of 0.36 in this specific test zone vs 0.06 for MICE-GBT. The results for RHOB are less convincing, 0.25 and 0.23 but the direct method can rely upon logs better suited to predicting RHOB which are available.

A single round of sequential BRR and KNN regression (MICE-O-BRR, MICEO-KNN) also outperformed direct imputation via D-GBT with values for DTS MSE of 0.06 and 0.05 respectively.

\subsection{Volve Log imputation error with increasing sparsity of input}

A key challenge to accurate imputation of well logs is the sparsity of the input. Sufficient training data is required to develop, calibrate and test a model. In this section we test the capabilities of the imputation models as sparseness is gradually increased in a random fashion to the input features. This is at odds with the often correlated missingess that occurs in individual wells but the limited size of the Volve data set necessitates this approach. On larger data sets a more blockly randomness could possible be pursued.

As sparsity increase there is an identifiable decrease in accuracy for all predictors. The change is more systematic when measuring the validation results as compared with the rest results (Figures 5). Also, the results for MICE imputers are very similar to the baseline once or direct approaches.

$\mathrm{BRR}$ continues to underperform reaching a critical point of failure at a sparsity fraction of 0.5 . The failure point for the other models tested appears at a sparsity fraction closer to 0.7. There is some jitter once BRR has failed which 
may be related to the distribution of missing values within zones.

The results when applying to the test data set were slightly different. At high sparsity the GBT-MICE model appears to outperform the D-GBT model. Breakdown of the models occurs around $50 \%$ sparsity.

\subsection{Volve Feature importance analysis}

The recent increase in the application of machine learning has also seen the development of techniques and methods designed to better explain the influence of input features on model outcomes. When there are a large number of input features it can be difficult to interpret why models behave the way they do and tailored workflows are required to establish causal links between input and output. One approach is the specialist algorithmic interpreter based upon Shapley Additive Explanations (SHAP) (Lundberg et al., 2020). SHAP uses game theory to assigns an impact score to a feature based upon the model output. Repeated stochastic testing of the model results in an overall view of how inputs affect the model output.

SHAP is well suited to explaining decision tree type algorithms and we apply SHAP here to our MICE-A-GBT model to better understand results and limitations of the model. To investigate the stability and importance of features during MICE iteration (specifically for the elastic target logs), we compare the second imputation round SHAP values to the last imputation round SHAP values (Figure 6). SHAP value swarm charts are generally interpretted by looking at the distribution of SHAP value impact. Input features with high importance are located at the top of the chart, they will have a large number of values distant from the origin (SHAP impact of zero) and cover a large range. The colour of the values indicates the direction in which the output feature is moved relative to the input feature. Features with less importance will cluster around the origin and have a small overall range. The number of clusters appearing along any features SHAP profile may indicate multiple distributions within a model (emphasising the need for non-parametric predictors).

For the MICE-A-GBT models the top three features tend to remain stable 
between the second and last imputation rounds. There is a reordering of the lesser features, but their impact values are often considerably smaller.

For the DT log, DTS, RHOB and NPHI are the top three logs in importance. The strong link between DT and DTS (the compressional and shear sonic slowness) is not surprising, but DTS is one of the least sampled logs in the Volve data set so its availability to inform DT is limited. RHOB and NPHI which are much more frequently sampled are likely contributing much of the information used to fill the DT missing values. Pseudo lithology logs like GR are evaluated by SHAP as having a very low impact upon the DT output.

DTS, one of the least sampled logs relies heavily on DT, this is the inverse relationship from previously. Most surprisingly, the ZONE (lithological formation) and deap resistivity logs (LogRD) occupy second and third position in importance. The ZONE SHAP values also operate in an inverse direction to the output values. This suggests that low value ZONES (shallow lithology) are increasing the output DTS whilst high value ZONE (deep lithology) are reducing DTS. In this case, ZONE may be acting as a proxy for depth, where natural compaction and increasing geological age generally decreases slowness. DTS is also more sensitive to rock competency than DT which is a complex function of mineral composition an micro-rock structure. Whilst the ZONE log does not inherently contain information about these properties it does group samples into common blocks that may aid with DTS prediction.

The RHOB models have the broadest reliance upon input features of the three target logs. DT, PEF, GR and NPHI all appear consistently with high impact.

In all cases. the features with the highest impact have a common distribution of SHAP values across imputation rounds indicating stability in the import and influence of key features during MICE iterations.

\subsection{Force 2020 Log Imputation}

For the FORCE 2020 data set we follow the same imputation procedure of train, validate and test that was applied to Volve data set. KNN type models 
Table 5: Metric scores for imputed values using different imputation algorithms on the F20 validation set. Best values are highlighted in bold.

\begin{tabular}{ccccccc}
\hline Model & Log & $\begin{array}{c}\text { Explained } \\
\text { Variance }\end{array}$ & $\begin{array}{c}\text { Maximum } \\
\text { Error }\end{array}$ & $\begin{array}{c}\text { Mean } \\
\text { Absolute } \\
\text { Error }\end{array}$ & $\begin{array}{c}\text { Mean } \\
\text { Squared } \\
\text { Error }\end{array}$ & $R^{2}$ \\
\hline MICE-BRR & DTS & 0.83 & 5.74 & 0.3 & 0.17 & 0.83 \\
MICE-O-BRR & DTS & 0.83 & 5.77 & 0.3 & 0.17 & 0.83 \\
MICE-A-GBT & DTS & 0.94 & 4.8 & 0.16 & 0.06 & 0.94 \\
MICE-R-GBT & DTS & 0.93 & $\mathbf{5 . 4 7}$ & 0.16 & 0.07 & 0.93 \\
D-GBT & DTS & $\mathbf{0 . 9 5}$ & 5.55 & $\mathbf{0 . 1 5}$ & $\mathbf{0 . 0 5}$ & $\mathbf{0 . 9 5}$ \\
\hline MICE-BRR & DT & 0.83 & 4.26 & 0.25 & 0.17 & 0.83 \\
MICE-O-BRR & DT & 0.83 & 4.3 & 0.25 & 0.17 & 0.83 \\
\hline MICE-A-GBT & DT & 0.95 & $\mathbf{3 . 1 6}$ & $\mathbf{0 . 1 1}$ & 0.05 & 0.95 \\
MICE-R-GBT & DT & 0.94 & 4.02 & $\mathbf{0 . 1 1}$ & 0.06 & $\mathbf{0 . 9 4}$ \\
D-GBT & DT & $\mathbf{0 . 9 6}$ & 3.43 & $\mathbf{0 . 1 1}$ & $\mathbf{0 . 0 4}$ & 0.96 \\
\hline MICE-BRR & RHOB & 0.7 & 10.2 & 0.39 & 0.3 & 0.7 \\
\hline MICE-O-BRR & RHOB & 0.74 & 5.56 & 0.37 & 0.26 & 0.74 \\
\hline MICE-A-GBT & RHOB & $\mathbf{0 . 9 1}$ & 5.38 & $\mathbf{0 . 2}$ & $\mathbf{0 . 0 9}$ & 0.91 \\
MICE-R-GBT & RHOB & $\mathbf{0 . 9 1}$ & $\mathbf{5 . 3 3}$ & $\mathbf{0 . 2}$ & $\mathbf{0 . 0 9}$ & 0.91 \\
\hline D-GBT & RHOB & $\mathbf{0 . 9 1}$ & 5.33 & $\mathbf{0 . 2}$ & $\mathbf{0 . 0 9}$ & $\mathbf{0 . 9 1}$ \\
\hline
\end{tabular}

were excluded from testing due to technical problems applying the method to the size of the data set.

All of the GBT type models again performed the best both in the training (Table 5) and testing (Table 6). The MICE-GBT models perform slightly better for DTS and much better for DT when compared with the D-GBT approach.

Qualitative analysis for the test results indicate a very good fit for the test data. In places, the BRR model is prone to generating large noise spikes in the DTS log (between samples 19,000 and 42,000, Figure 7). The noise spikes don't appear to be associated with any particular missing log and are perhaps due to a lack of training in a particular zone. Comparatively, the GBT logs show a consistently good fit outperforming the other models and validating the quantitative metric results. The fit to the long wavelength variations is particularly strong.

An analysis of the relationship between metric based prediction performance 
Table 6: Metric scores for imputed values using different imputation algorithms on the F20 test set. Best values are highlighted in bold.

\begin{tabular}{ccccccc}
\hline Model & Log & $\begin{array}{c}\text { Explained } \\
\text { Variance }\end{array}$ & $\begin{array}{c}\text { Maximum } \\
\text { Error }\end{array}$ & $\begin{array}{c}\text { Mean } \\
\text { Absolute } \\
\text { Error }\end{array}$ & $\begin{array}{c}\text { Mean } \\
\text { Squared } \\
\text { Error }\end{array}$ & $R^{2}$ \\
\hline MICE-BRR & DTS & 0.89 & 2.44 & 0.22 & 0.09 & 0.89 \\
MICE-O-BRR & DTS & 0.89 & 2.4 & 0.23 & 0.09 & 0.89 \\
MICE-A-GBT & DTS & 0.92 & 2.59 & 0.18 & 0.07 & 0.92 \\
MICE-R-GBT & DTS & $\mathbf{0 . 9 4}$ & 2.54 & $\mathbf{0 . 1 6}$ & $\mathbf{0 . 0 5}$ & $\mathbf{0 . 9 4}$ \\
D-GBT & DTS & 0.93 & $\mathbf{2 . 6 5}$ & 0.18 & 0.06 & 0.93 \\
\hline MICE-BRR & DT & 0.88 & 2.16 & 0.21 & 0.1 & 0.88 \\
MICE-O-BRR & DT & 0.87 & 2.25 & 0.2 & 0.1 & 0.87 \\
MICE-A-GBT & DT & $\mathbf{0 . 9 4}$ & 2.08 & $\mathbf{0 . 1 6}$ & $\mathbf{0 . 0 5}$ & $\mathbf{0 . 9 4}$ \\
MICE-R-GBT & DT & 0.91 & $\mathbf{3 . 0 1}$ & 0.17 & 0.07 & 0.91 \\
D-GBT & DT & 0.88 & 2.22 & 0.19 & 0.1 & 0.87 \\
\hline MICE-BRR & RHOB & 0.72 & 4.82 & 0.37 & 0.26 & 0.72 \\
\hline MICE-O-BRR & RHOB & 0.77 & 4.18 & 0.34 & 0.21 & 0.77 \\
\hline MICE-A-GBT & RHOB & 0.86 & 4.01 & 0.25 & 0.13 & 0.86 \\
MICE-R-GBT & RHOB & $\mathbf{0 . 8 7}$ & $\mathbf{3 . 8 3}$ & $\mathbf{0 . 2 4}$ & $\mathbf{0 . 1 2}$ & $\mathbf{0 . 8 7}$ \\
\hline D-GBT & RHOB & $\mathbf{0 . 8 7}$ & 4.0 & $\mathbf{0 . 2 4}$ & $\mathbf{0 . 1 2}$ & $\mathbf{0 . 8 7}$ \\
\hline
\end{tabular}


and the number of samples available for training within each geological formation (Figure 8) show erratic trends in performance when the training sample size is small. As the number of samples in a geological zone increases beyond approximately 20,000 points both MAE and MSE metrics trend towards a more stable value. Trends in $R^{2}$ and explained variance are less clear.

\section{Discussion}

\subsection{Explanation of Results}

On the whole, multiple iterations of the MICE algorithm do not appear to improve the overall predictive capacity of the models implemented. There are exceptions where certain combinations of missing logs and sequential imputation and prediction are desirable but this does not appear to form the bulk of missing values in the tested data sets. GBT generally out performed the BRR and KNN models. The ability for GBT to handle missing values is a significant advantage with performance as good or better than the MICE-GBT method.

The exceptions where MICE improves upon direct imputation were observed when the elastic logs were all missing. In these scenarios complete prediction of all three targets is required and accuracy improves when sequential imputation is performed. In these cases, the more complex non-parametrics replations between logs are handled better. For example, DRHO may be used to predict RHOB which is subsequently used to predict DT and then DTS.

Although MICE is relatively inexpensive for log data it is computationally more expensive than direct or single pass sequential imputation. MICE typically runs for 10-20 iterations requireing multiple imputation predictors to be trained and stored.

Of the three machine learning predictive models tested, GBT appears to be a clear leader, exhibiting superior correlation and lower error. The strength of the ensemble approach over BRR and KNN may reflect a superior capacity to identify and model the complex non-parametric relationships between logs.

An observation from both data sets was the relative under performance of models in geological zones with small sample sizes, which was to be expected. A 
subsequent analysis calculating metric scores for each zone (Figure 8), identified significant variations in metric scores for the test logs when sample sizes are small. We attribute the variance in the scores at small sample size to complex and multi-factored interactions between the available training samples, the sparsity of the input features for the zone and the effectiveness of cross-training between lithologically similar zones. The zones with larger sample sizes tend to exhibit more stable metric behaviour leading us to suspect insufficient data both for training purposes, and for the data available to calculate representative metric scores at the zone scale.

At larger sample sizes, there is a compression in the error variance across zones (particularly for the error metrics MSE/MAE) which may provide indicators for the number of samples needed in a zone to achieve some stability in the prediction model.

A more robust approach over using geological zones may be to consider undertaking this analysis based upon lithological characteristics rather than formation names. This could be achieved either via manual labelling, grouping of similar zone labels or, if the sample set is sufficiently dense, automated clustering.

Empirical models for elastic logs rely upon and emphasise their strong correlations. The SHAP analysis of the GBT approach confirms the interdependence of the elastic input features for accurate prediction (even with non-parametric methods) but intrinsically extends them to include other features as further control upon the model output. For example, while typical empirical workflows for prediction of compressional sonic from density would require additional corrections for depth and fluid content, the GBT model can leverage other input features such as sample depth and the resistivity to form better predictions. This greatly simplifies the entire workflow, and we would recommend utilising GBT for imputation.

\subsection{MICE limitations and assumptions}

All imputation or prediction methodologies rely upon a sufficient quantity of data to correctly calibrate the model and MICE is no different. If characteristi- 
cally unique sections of log are missing from the training data, the predictive models will be unable to reproduce such data with any accuracy. This limits the application of MICE to data sets with representative sampling but is a common problem to all parts of machine learning; the machine cannot model what it has not seen. This leads to poor generalisation of the model unless the training data set is sufficiently diverse. In practice, and for well log imputation, the pragmatic approach would be to tailor an imputation model for each unique input data set. The cost of training, at least with the models tested was acceptably low (on the order of minutes), but for more costly prediction models further consideration may be required.

There is also a degree of non-repeatability with most ML predictors and therefore any application of MICE will be limited by the chosen prediction algorithm. If the input data set is augmented or changed, the output predictions and imputations are also likely to change. The degree of difference observed will depend upon the degree of changes to the input and the dependence upon randomness in the training of the prediction models. The non-repeatable nature of the imputations may discourage downstream users of the data who require stable logs as input to their own workflows. In these cases the general prediction capability of ML regression models must be traded off against the labour intensive but more stable empirical or manual prediction approach. A possible solution could be to capture and store multiple imputed versions as a measure of the imputation uncertainty in a manner similar to but distinct from Diaz and Zadrozny (2020).

\subsection{Hyperparameter Tuning}

MICE has relatively few hyperparameters; maximum iterations, convergence tolerance and imputation order. For this study, imputation order, either random or ascending did not appear to greatly influence the results and maximum iterations and convergence tolerance were left at their default values.

MICE-GBT and the direct GBT were together the best prediction models tested in this study. Both were submitted to a four hyperparameter tuning grid 
search that varied the minimum leaf size, the maximum tree depth and the bagging frequency and fraction.

In general it was found that the bagging parameters had little effect upon the overall results although very low bagging fractions $(<0.2)$ tended to cause training issues and should be avoided. There was no benefit to the prediction metrics when the maximum depth of the trees was set at greater than 7. Larger tree depths should probably be avoided to prevent over fitting. Increasing the minimum leaf size tended to decrease the maximum error, preventing out-liers in the prediction but at the expense of over generalising the solution and in turn increase the mean squared error. From the metrics, the optimum minimum leaf size was determined to be around 300 for the MICE implementation and 500 for the direct method.

Comparisons of the base and tuned model outputs demonstrated little to no improvement for the direct prediction models and some improvement when using tuned parameters within the MICE methods. Some instability in the result was observed when a minimum leaf size of 300 was used for the iterative method and reducing this to 200 greatly improved the results. The improvements in prediction accuracy for the MICE models were most notable in the RHOB log results where a bias in the result is removed.

\subsection{Further research}

Although the authors would recommend the use of MICE or indeed direct imputation using the machine learning models tested, there are additional tests that may improve our application and understanding of MICE for well log prediction and imputation. For training validation, we use randomised selection of points within logs. This is not typically how gaps occur in well log data. Alternative approaches such as the method employed by Lopes and Jorge (2018) of pseudo modelling the gaps may further test the robustness of our approach. Validation tests could also be augmented to check for over-training by utilising $\mathrm{k}$-fold cross-validation methods common in machine learning.

We also suggest that the MICE algorithm might be modified to improve 
and or automate noise or bad data rejection. Currently, log editing is required beforehand to quality control the input, the MICE process may be a tool that can identify and automatically remove data which fails a tolerance criterion when compared with predictions. Initial imputation values use by MICE methods could also be improved by using empirical relationships rather than the mean value of a feature.

Unlike Brown et al. (2020), we have not included derived petrophysical logs in this study. From a machine learning perspective, petrophysical logs such as water saturation, porosity and clay volume can be viewed as engineered features which augment or extend our view of the raw input data. Their addition to the imputation workflow may improve correlations and relationships between the raw data that were ignored previously. Future tests may consider using these data as input if available. Similar to the work of Brown et al. (2020), this would also result in imputed petrophysical products.

There are many ML algorithms and we have tested some of the easiest to implement. Deep learning such as convolutional neural networks which can better account for adjacency in samples may benefit from the MICE approach to imputation. Indeed, most ML methods cannot handle missing data so iterative imputation may improve these models.

\section{Conclusion}

Many subsurface analysis tasks and workflows rely upon or can benefit from a complete well logging data set. However, in many cases the logging measurements are rarely complete with gaps or logs missing entirely. This study has utilised the MICE approach to successfully and completely impute multiple well logs simultaneous using ML algorithms. Of the four algorithms that were tested, gradient boosted trees performed the best. Although MICE did not always improve the directed imputation of logs when using GBT, imputation when certain combinations of missing logs are missing may benefit from the iterative approach. MICE can also improve GBT results when the sparsity of the input data is high. 
Finally, while GBT have the ability to naturally handle missing values in the input features, many ML algorithms cannot. MICE may prove more useful in scenarios where algorithms require complete input features for training.

\section{Computer code and data availability}

Source code used for analysis and log imputation using MICE is available from the first author and can be downloaded from https://github.com/trhallam/ mice_well_log_imputation

THe Volve well log data is available for download from the Volve Data Village provided by Equinor at https://www.equinor.com/en/what-we-do/ digitalisation-in-our-dna/volve-field-data-village-download.html.

11 The Force 2020 data is available for download from Xeek https://xeek.ai/ challenges/force-well-logs/overview

\section{Declaration of competing interest}

The authors declare that they have no known competing financial interests or personal relationships that could have appeared to influence the work reported in this paper.

\section{Authorship statement}

Antony Hallam developed the methodology, the code, worked on the application and the writing; Debajoy Mukherjee developed the code and methodology; Romain Chassagne discussed the methodology, supervised the research and writing.

\section{Acknowledgements}

We would like to thank the sponsors of the Edinburgh Time-Lapse Project, Phase VII: AkerBP, BP, CGG, Chevron/Ithaca Energy CNOOC, Equinor, ConocoPhillips, ENI, Petrobras, Norsar, Woodside, Taqa, Halliburton, ExxonMobil, OMV and Shell for financial support. Equinor for provision of the Volve public dataset. The FORCE 2020 sponsors and the Norwegian Government for the 
F2020 dataset. Thank you to colleagues and peers for their help and feedback. We also acknowledge Schlumberger for the use of their software and the Python open source community.

\section{References}

Azur, M. J., Stuart, E. A., Grangakis, C., and Leaf, P. J. (2011). Multiple imputation by chained equations: what is it and how does it work? International Journal of Methods in Psychiatric Research, 20(1):40-49.

Bormann, P., Aursand, P., Dilib, F., and Dischington, P d Manral, S. (2020). 2020 FORCE Machine Learning Contest.

Brown, N., Roubíčková, A., Lampaki, I., MacGregor, L., Ellis, M., and Vera de Newton, P. (2020). Machine learning on Crays to optimize petrophysical workflows in oil and gas exploration. Concurrency Computation, 32(20).

Bukar, I., Adamu, M. B., and Hassan, U. (2019). A machine learning approach to shear sonic log prediction. Society of Petroleum Engineers - SPE Nigeria Annual International Conference and Exhibition 2019, NAIC 2019.

Christ, M., Braun, N., Neuffer, J., and Kempa-Liehr, A. W. (2018). Time series feature extraction on basis of scalable hypothesis tests (tsfresh - a python package). Neurocomputing, 307:72 - 77 .

Churikov, S. N. and Grafeeva, N. (2018). Recovering gaps in the gamma-ray logging method. International Multidisciplinary Scientific GeoConference Surveying Geology and Mining Ecology Management, SGEM, 18(2.2):361-368.

Diaz, J. L. G. and Zadrozny, B. (2020). Propagation of Well-Log Data Imputation Uncertainties Towards the Interpolated 3D-Petrophysical Map Using Epistemic Kernels and Kriging. In EAGE 82nd Conference and Exhibition, number December 2020, Amsterdam, Netherlands. EAGE. 
Dramsch, J. S. (2020). Chapter one - 70 years of machine learning in geoscience in review. In Moseley, B. and Krischer, L., editors, Machine Learning in Geosciences, volume 61 of Advances in Geophysics, pages 1-55. Elsevier.

Dubey, H. and Pudi, V. (2013). Cluekr : Clustering based efficient knn regression. In Pei, J., Tseng, V. S., Cao, L., Motoda, H., and Xu, G., editors, Advances in Knowledge Discovery and Data Mining, pages 450-458, Berlin, Heidelberg. Springer Berlin Heidelberg.

Feng, R., Grana, D., and Balling, N. (2021). Imputation of missing well log data by random forest and its uncertainty analysis. Computers $\& 6$ Geosciences, 152(March):104763.

Freund, Y. and Schapire, R. E. (1997). A Decision-Theoretic Generalization of On-Line Learning and an Application to Boosting. Journal of Computer and System Sciences, 55(1):119-139.

Gardner, G. H. F., Gardner, L. W., and Gregory, A. R. (1974). Formation Velocity and Density - The diagnostic for stratigraphic traps. Geophysics, $39(6): 770-780$.

Ghaithi, A. A. (2020). Deep Learning Methods for Shear Log Predictions in the Volve Field Norwegian North Sea. Master of science (geophysical engineering), Colorado School of Mines.

Greenberg, M. L. and Castagna, J. P. (1992). Shear-wave velocity estimation in porous rocks: theoretical formulation, preliminary verification and applications. Geophysical Prospecting, 40(2):195-209.

Hallam, T., MacBeth, C., Chassagne, R., and Amini, H. (2020). 4D seismic study of the Volve Field - an open subsurface-dataset. First Break, 38(2):59-70.

Hampson, D. P., Schuelke, J. S., and Quirein, J. A. (2001). Use of multiattribute transforms to predict log properties from seismic data. Geophysics, 66(1):220236. 
Hegde, C. and Gray, K. E. (2017). Use of machine learning and data analytics to increase drilling efficiency for nearby wells. Journal of Natural Gas Science and Engineering, 40:327-335.

James, G., Witten, D., Hastie, T., and Tibshirani, R. (2013). An Introduction to Statistical Learning with Applications in R. Springer-Verlag, New York, 7 edition.

Jian, H., Chenghui, L., Zhimin, C., and Haiwei, M. (2020). Integration of deep neural networks and ensemble learning machines for missing well logs estimation. Flow Measurement and Instrumentation, 73:101748.

Ke, G., Meng, Q., Finley, T., Wang, T., Chen, W., Ma, W., Ye, Q., and Liu, T. Y. (2017). LightGBM: A highly efficient gradient boosting decision tree. Advances in Neural Information Processing Systems, 2017-December(Nips):3147-3155.

Lopes, R. L. and Jorge, A. M. (2018). Assessment of predictive learning methods for the completion of gaps in well log data. Journal of Petroleum Science and Engineering, 162(November):873-886.

Lundberg, S. M., Erion, G., Chen, H., DeGrave, A., Prutkin, J. M., Nair, B., Katz, R., Himmelfarb, J., Bansal, N., and Lee, S.-I. (2020). From local explanations to global understanding with explainable AI for trees. Nature Machine Intelligence, 2(1):56-67.

Lundberg, S. M. and Lee, S.-I. (2017). A unified approach to interpreting model predictions. In Guyon, I., Luxburg, U. V., Bengio, S., Wallach, H., Fergus, R., Vishwanathan, S., and Garnett, R., editors, Advances in Neural Information Processing Systems 30, pages 4765-4774. Curran Associates, Inc.

Murray, J. S. (2018). Multiple imputation: A review of practical and theoretical findings. Statistical Science, 33(2):142-159.

Poloczek, J., Treiber, N. A., and Kramer, O. (2014). Knn regression as geoimputation method for spatio-temporal wind data. In de la Puerta, J. G., 
Ferreira, I. G., Bringas, P. G., Klett, F., Abraham, A., de Carvalho, A. C., Herrero, Á., Baruque, B., Quintián, H., and Corchado, E., editors, International Joint Conference SOCO'14-CISIS'14-ICEUTE'14, pages 185-193, Cham. Springer International Publishing.

Raghunathan, T. E., Lepkowski, J. M., Hoewyk, J. V., and Solenberger, P. (2001). A Multivariate Technique for Multiply Imputing Missing Values Using a Sequence of Regression Models. Survey Methodology, 27(1):85-95.

Russell, B. H., Hampson, D. P., and Lines, L. R. (2003). Application of the radial basis function neural network to the prediction of log properties from seismic attributes - A channel sand case study. 2003 SEG Annual Meeting, pages $1-4$.

Sarkar, D., Bali, R., and Sharma, T. (2018). Practical Machine Learning with Python. Springer Science, New York.

Tipping, M. E. (2001). Sparse Bayesian Learning and the Relevance Vector Machine. Journal of Machine Learning Research, 1(3):211-244.

Tittman, J. (1986). Geophysical well logging. page 186.

van Buuren, S. (2007). Multiple imputation of discrete and continuous data by fully conditional specification. Statistical Methods in Medical Research, 16(3):219-242.

van Buuren, S. and Groothuis-Oudshoorn, K. (2011). mice: Multivariate imputation by chained equations in R. Journal of Statistical Software, 45(3):1-67.

Varoquaux, G., Buitinck, L., Louppe, G., Grisel, O., Pedregosa, F., and Mueller, A. (2015). Scikit-learn. GetMobile: Mobile Computing and Communications, 19(1):29-33. 
(a) Log fraction missing by well.

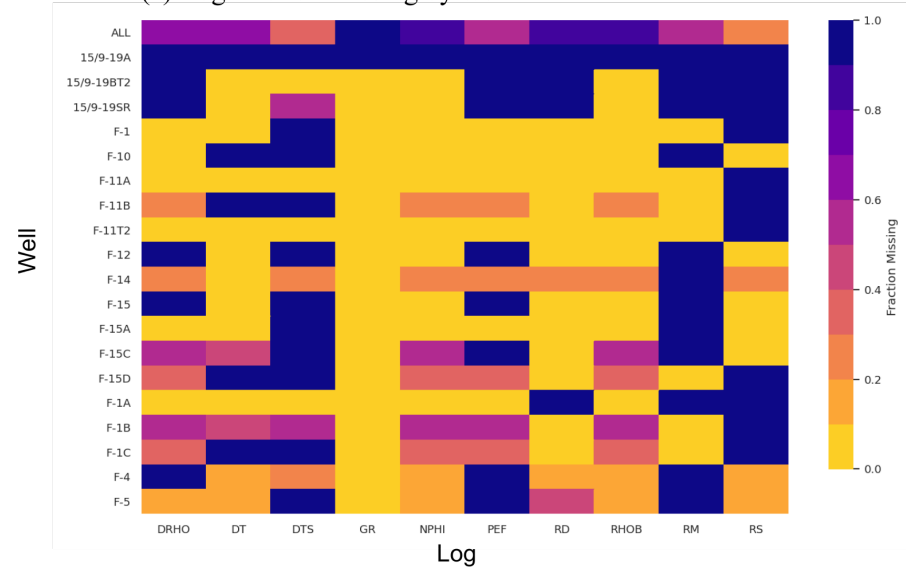

(b) Log fraction missing by stratigraphic zone.

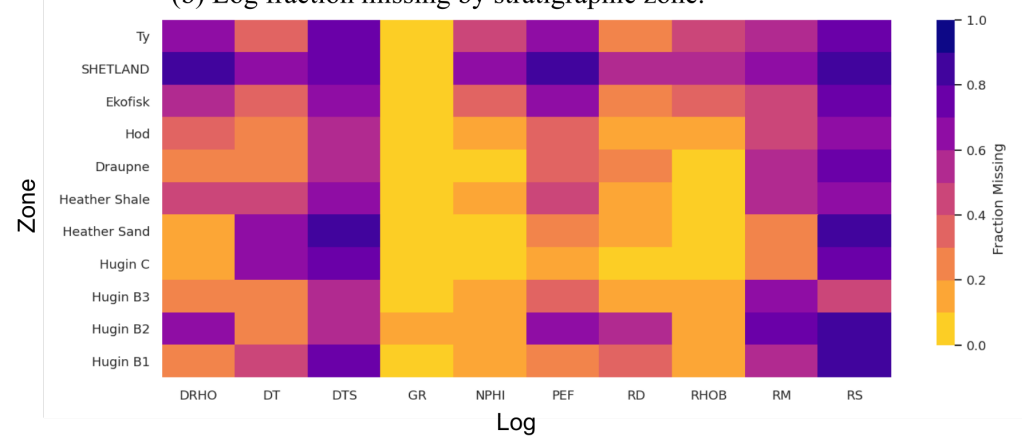

(c) Missing data map by log.

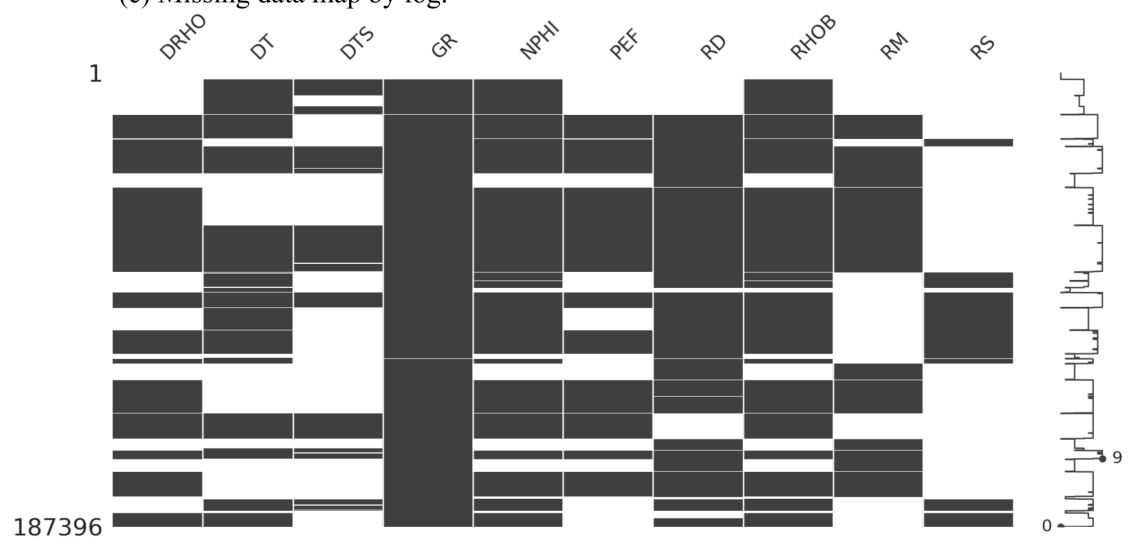

Figure 1: Missing data characterisation for Volve data set. (c) white is for missing data 
(a) Log fraction missing by well

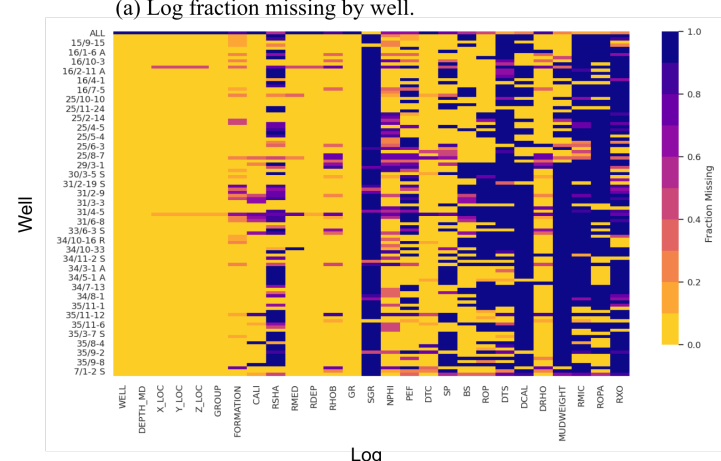

(b) Log fraction missing by stratigraphic zone.
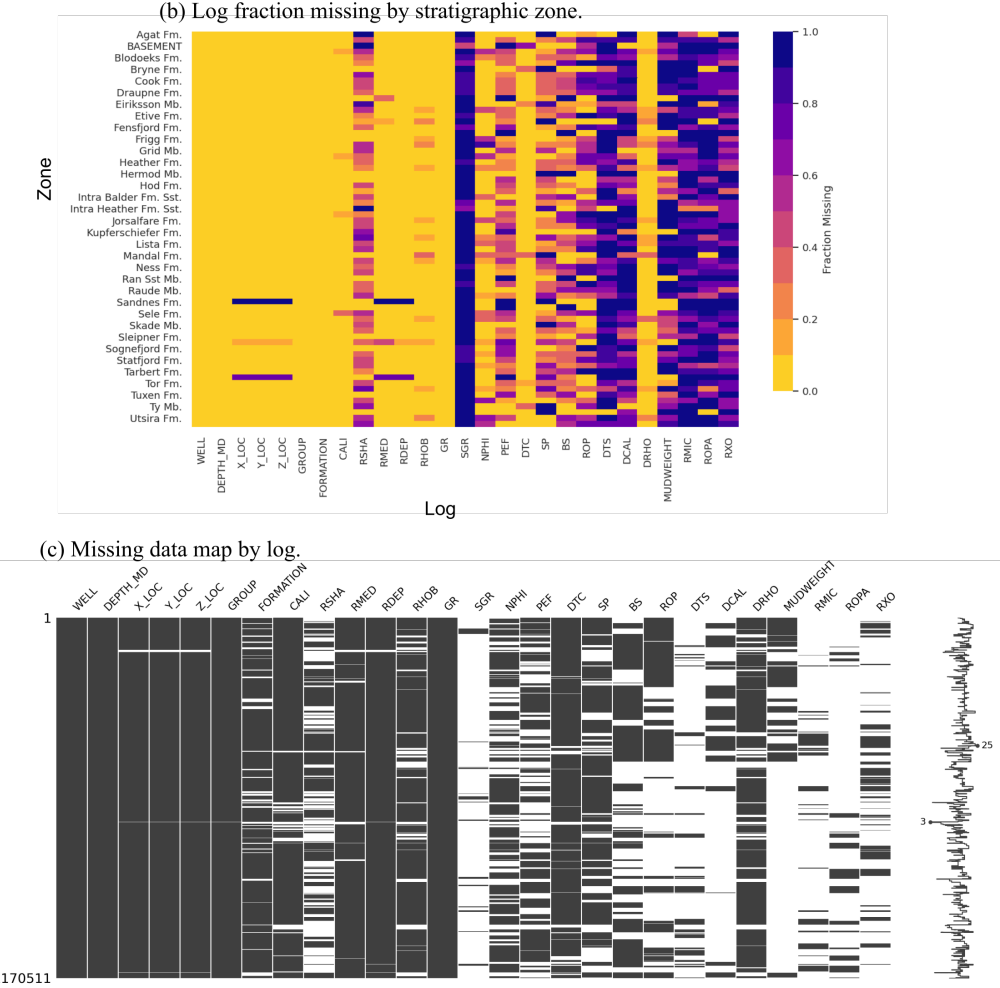

Figure 2: Missing data characterisation for Force 2020 and Volve data set. 

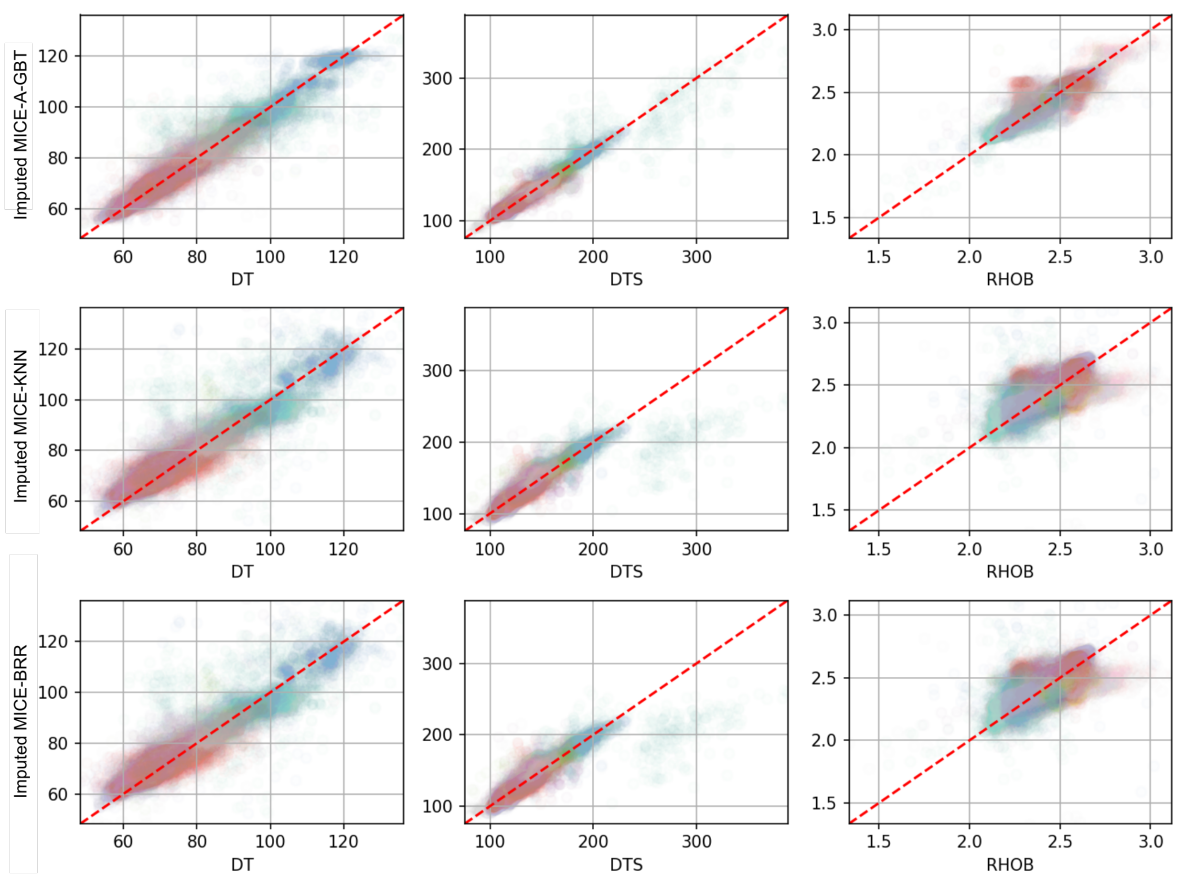

Figure 3: Recorded versus Imputed Results for DT, DTS and RHOB logs using MICE-A-GBT (top) MICE-KNN (middle) and MICE-BRR (bottom). Points are coloured by stratigraphic zone. Intensity of colour corresponds to the density of samples. 

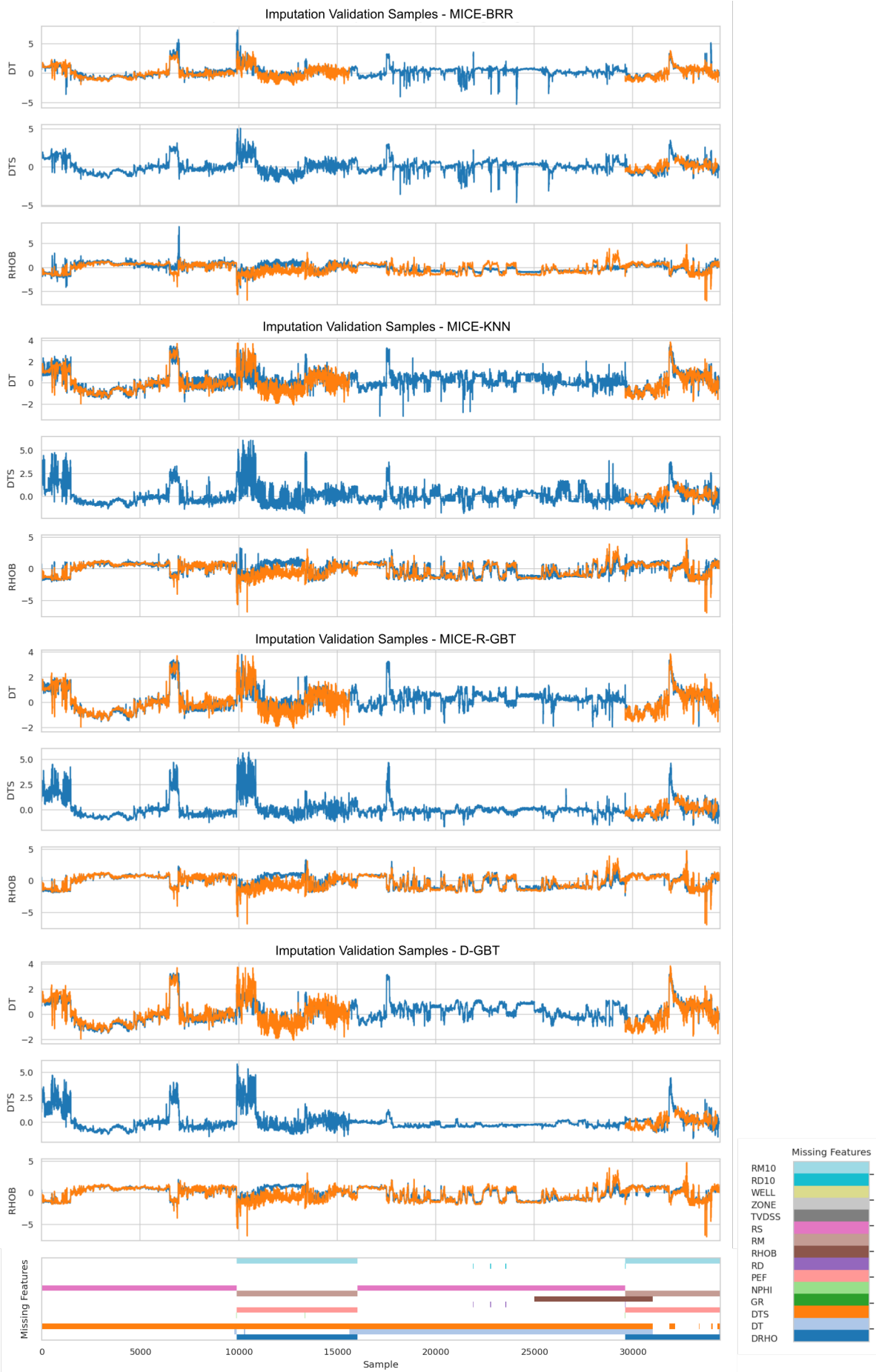

Figure 4: Test data imputation and prediction with the four tested imputation models. Imputed values are in blue and true values in orange. The bottom frame shows missing values of the input features prior to imputation. 
(a)

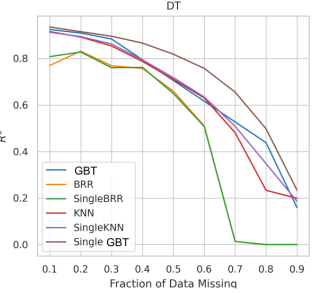

(b)

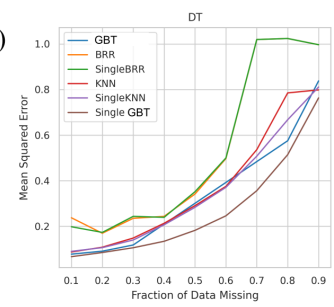

(c)

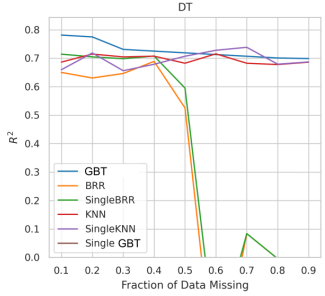

(d)

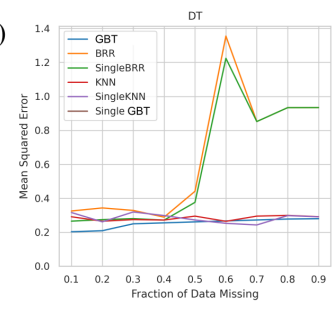

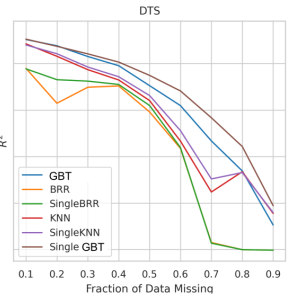

DTS
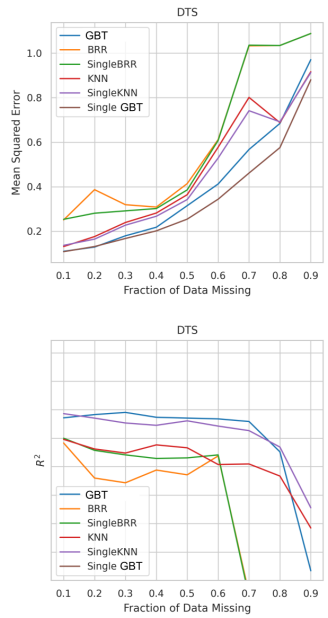

DTS

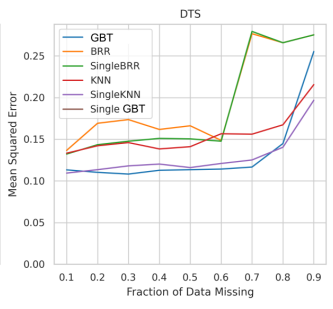

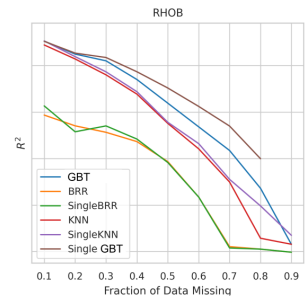

Rнов
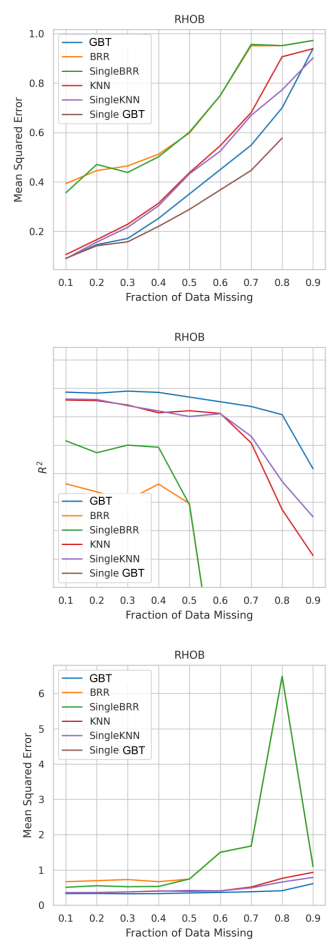

Figure 5: Volve test results for increasing sparsity of input data; (a, b) $R^{2}$ and MSE for validation data, (c, d) $R^{2}$ and MSE for test data. 
(a)

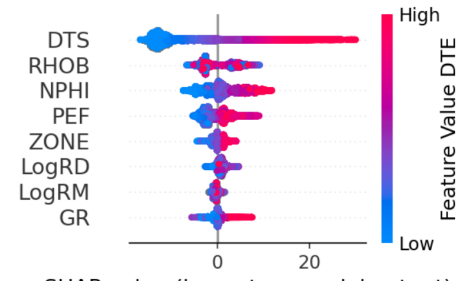

SHAP value (impact on model output)

(c)

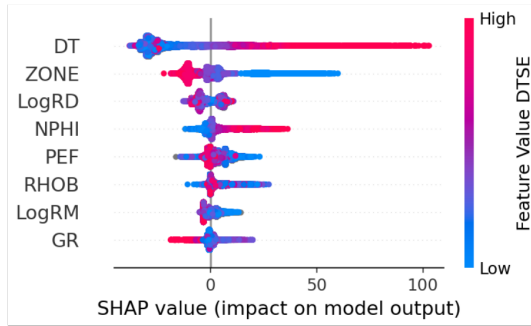

(e)

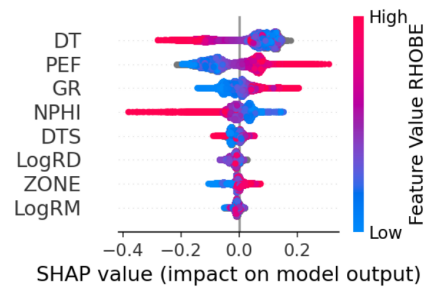

(b)

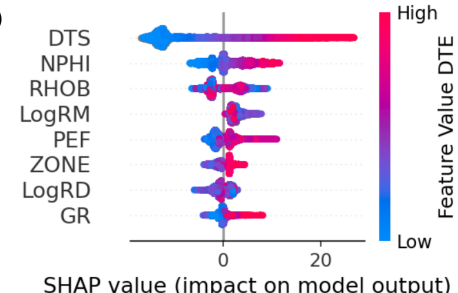

(d)

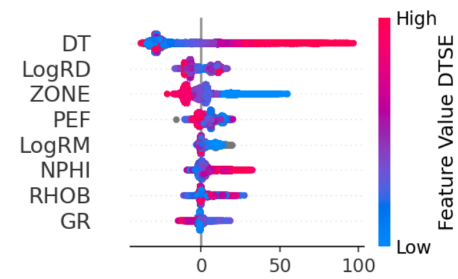

SHAP value (impact on model output)

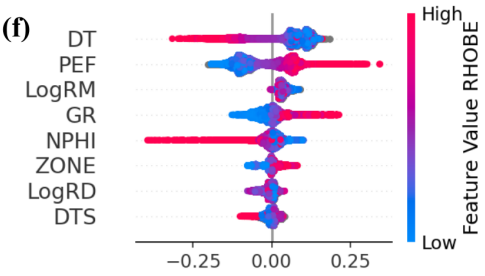

SHAP value (impact on model output)

Figure 6: SHAP Values for second and last imputation rounds of MICE-A-GBT models for (a, b) DT, (c, d) DTS, and (e, f) RHOB. 


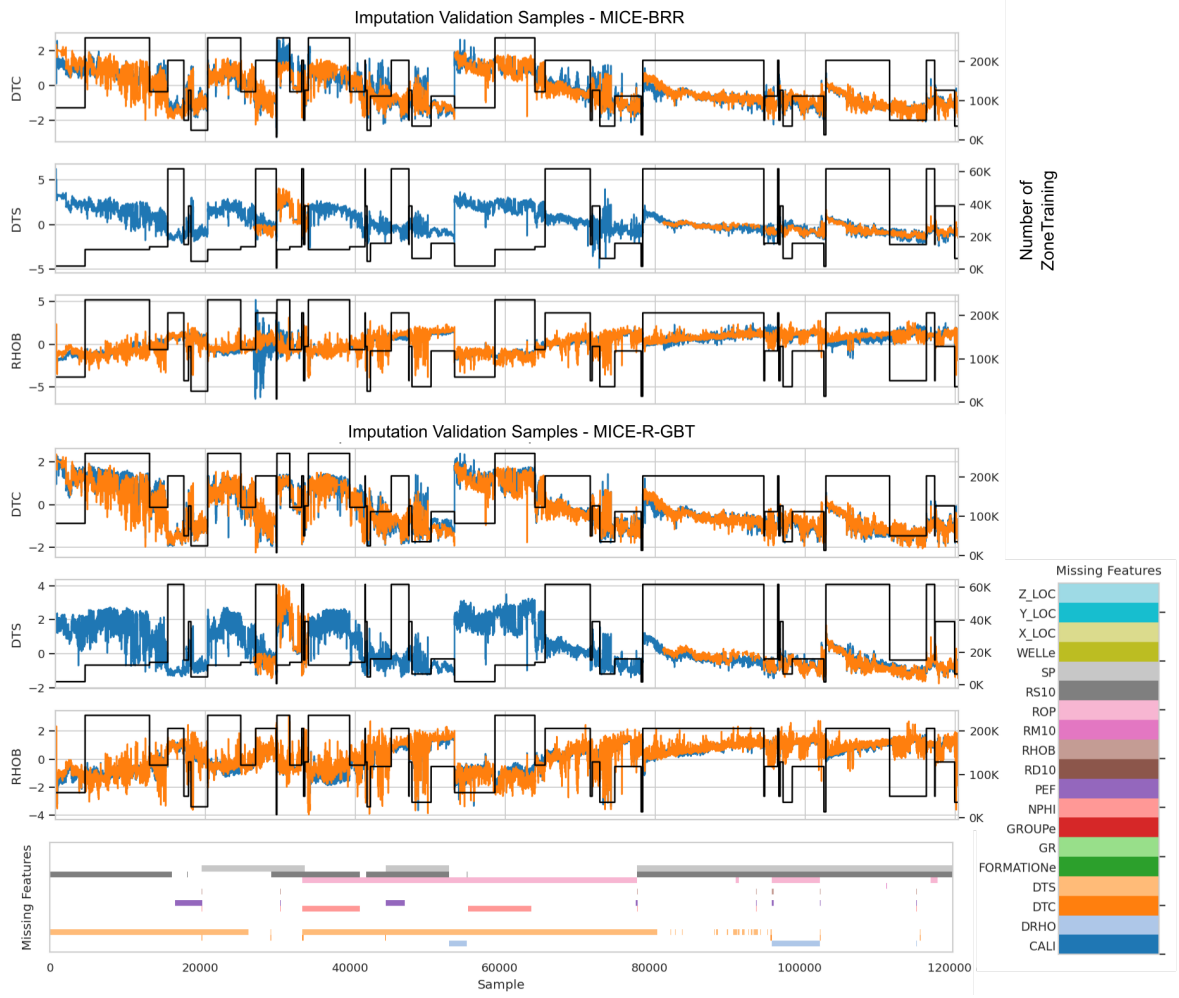

Figure 7: Force 2020 test data imputation and prediction with the MICE-BRR and MICE-GBT model. Imputed values are in blue and true values in orange. The bottom frame shows missing values of the input features prior to imputation and the black line (right axis) indicates the number of samples available for training in that geological super group. 

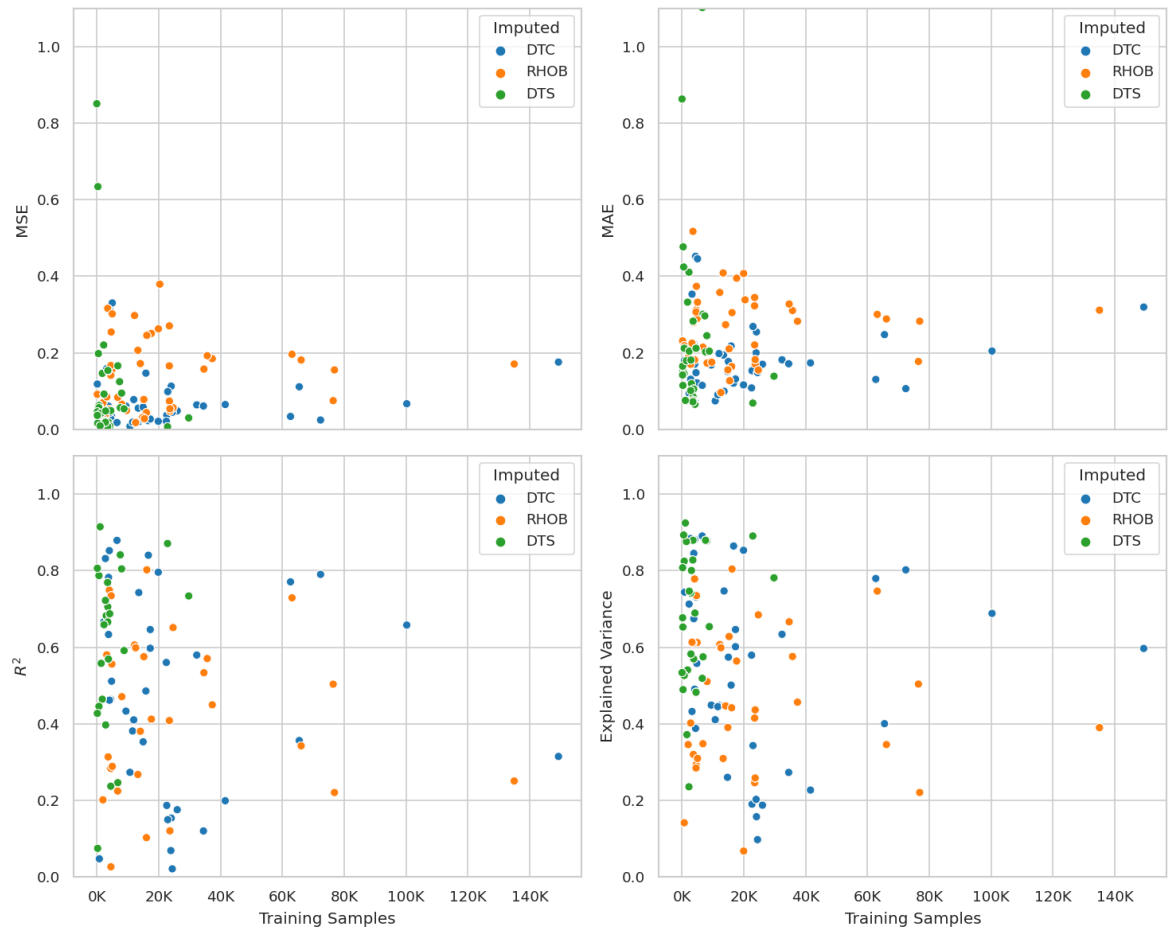

Figure 8: Force 2020 error metrics plotted by imputed target and geological formation for the MICE-A-GBT model. 